\title{
LAS «REFORMAS» DE URUKAGINA
}

\author{
Manuel Molina \\ (Universidad de Murcia)
}

\section{SUMMARY}

In this article a study is made of the Sumerian text known as The "Reforms" of UruKAgina, taking into consideration aspects such as the composition of the text, problems related to its translation, the contents and the real significance of "The Reforms", and the social, economical, political and ideological context in which it was promulgated. Also a transliteration and translation of The "Reforms"... (UKg 4-5 = cones B-C) are provided.

\section{EL SOPORTE DEL TEXTO}

Es bien sabido que la mayoría de los muchos miles de textos cuneiformes que nos han legado los diferentes pueblos que habitaron la Antigua Mesopotomia se redactaron sobre tablillas de arcilla. Habitualmente se ha considerado que estos documentos, en cuanto que objetos, proporcionan escasa información que añadir a la del contenido del propio texto.

Este no es el caso, sin embargo, de las llamadas «inscripciones reales», de contenido celebrativo o votivo. Dichas inscripciones - que también podían realizarse sobre tablillas de arcilla- se redactaban en ocasiones sobre cilindros, conos $u$ otros artefactos destinados a ser enterrados bajo los muros o pavimentos de los edificios cuya construcción se conmemoraba (no estaban dirigidos, por tanto, a la lectura pública)', o bien sobre objetos preciosos de carácter votivo. Así, en estos textos la identificación de su soporte serviría para comprender las motivaciones de su redacción y, por consiguiente, también para precisar las implicaciones de su contenido.

1 Cf. R.S. Ellis, Foundation Deposits in Ancient Mesopotamia, Yale Near Eastern Researches 2, New Haven Londres 1968. 
En lo que concierne al texto de Las «Reformas» de UruKAgina (c. 2350 a.C.), un estudio de J.S. Cooper ${ }^{2}$, realizado sobre inscripciones reales presargónicas, ha contribuido a aclarar algunas de las cuestiones no resueltas por R.S. Ellis.

Las «Reformas»... se han conservado en ocho inscripciones que contienen tres versiones diferentes del mismo texto ${ }^{3}$ :

I: Ukg I $\quad($ MNB 1390, Girsu $)=$ Cono A: (AO 27621, Girsu):

Ukg $2 \quad($ AO 12181, Girsu $)=$ Cono Aa: Ukg 3 (AO 12782, Girsu) = Cono Ab: Ukg 62 (IM 5642, ?):

II: Ukg 4 (AO 3278, Girsu) = Cono B: Ukg 5 (AO 3149, Girsu) = Cono C:

Ukg 60 (CTS 5, ?) = Cono D:

III: Ukg 6 (ESEM 1717, Girsu): jarra o cono realizado a torno. jarra o cono realizado a mano? . objeto esferoide? realizado a torno. jarra o cono realizado a torno. ? cono realizado a mano (técnica en anillo). cono realizado a mano (técnica en anillo). ?

placa oval de arcilla.

Como puede observarse, no es seguro que la versión I de Las «Reformas»... se hubiese redactado sobre los tradicionales conos de fundación. De haber sido otro el soporte (jarras), la función del texto habría sido diferente y, por ello, también habrá de serlo nuestro análisis del mismo. Curiosamente, lo mismo ocurre con los llamados textos «históricos» que narran el conflicto que enfrentó a las ciudades de Lagaš y de Umma, algunos de los cuales se escribieron con certeza sobre jarras.

Por otra parte, las características físicas de los soportes de las inscripciones de esta I Dinastía de Lagaš que sí pueden ser descritos como conos son ciertamente peculiares: el cono $\mathrm{A}$ de Entemena (Ent 28) y los conos B y C de UruKAgina (Ukg 4 y 5) miden alrededor de $27 \mathrm{~cm}$ de alto, y los diámetros de sus bases oscilan entre $12-16 \mathrm{~cm}$; los conos de UruKAgina están realizados a mano (técnica en anillo), con pequeñas perforaciones en la punta y una gran abertura en la base; el cono de Entemena está hecho a torno, excepto en su tercio superior, no presenta perforación en la punta y, a diferencia de los otros conos, se lee desde la base hacia arriba.

2 J.S. Cooper, «Medium and Message: Inscribed Clay Cones and Vessels from Presargonic Sumer», Revue d'Assyriologie 79 (1985) 97-114.

3 La numeración de los ejemplares (entre paréntesis se ofrece el $n^{\circ}$ de museo y la procedencia) corresponde a la establecida por E. Sollberger (Corpus des Inscriptions "Royales» Présargoniques de Lagash, Genève 1956), después proseguida por H. Steible (Die alsstmerischen Bau- und Weihinschrifien I-II, Freiburger Altorientalische Studien 5, Wiesbaden 1982). La información sobre el tipo de objeto y la técnica de fabricación proviene de J.S. Cooper «Medium and Message...», p. 98, table 1.

De aquí en adelante, todas las referencias al texto de Las «Reformas» de UruKAgina se harán sobre la version II (var. Ukg 5), la más completa de las tres, transliterada y traducida en la segunda parte de este artículo. A las mencionadas ediciones de E. Sollberger y H. Steible corresponden las citas de los textos abreviados como: Ean (= Eanatum [Lagaš]), Eig (= E'iginimpa'e [Adab]), En I (= Enanatum I [Lagaš]), En II (= Enanatum II [Lagas]), Ent (= Entemena [Lagaš]), Enz (= Enentarzi [Lagaš]), Lug (= Lugalanda [Lagaš]), Lugd (= Lugaldalu [Adab]), Lukis (= Lugalkisalsi [Uruk]), Lum (= Lumma [Adab]), LuTAR (= Lugal-TAR [Uruk]), Luzag (= Lugalzagesi [Umma-Uruk]), Med (= Medurba [Adab]), Mes (= Mesilim [Kiš $]$ ), Uhb (= Uhub [Kiš $]$ ), Ukg (= UruKAgina [Lagaš]), Url (= Urlunma [Umma]), Uin (= Ur-Nanše [Lagaš]).

Los años de reinado de UruKAgina como lugal, una vez que abandona el título de ensi, se abrevian Ul $n$.

4 Fragmento inicialmente considerado como parte de Ukg 1. Cooper («Medium and Message ...», pp. 99, 105), sin embargo, ha puesto en duda esta identificación. 
Así, por su forma y contenido, estos conos y jarras se distancian notablemente de los típicos depósitos de fundación. Su verdadera función, sin embargo, está todavía lejos de poder aclararse: la tímida propuesta de Cooper de relacionar estas inscripciones con los posteriores kudurru's (estelas fronterizas) podría quizás adecuarse a los textos de Eanatum y Entemena que narran el conflicto entre Lagaš y Umma, pero difícilmente a los de las Las «Reformas» de UruKAgina.

\section{LA TRADUCCIÓN DEL TEXTO}

La inscripción, de considerable longitud, presenta, como es lógico, algunas dificultades de traducción. Estos problemas filológicos (que en su aspecto más específico se detallan en las notas a la traducción del texto) son los derivados de tres cuestiones que afectan también a la inmensa mayoría de la documentación mesopotámica sumeria:

1) Nuestro conocimiento de la paleografía cuneiforme, si bien muy avanzado, adolece todavía de algunos problemas. La escritura cuneiforme, como es sabido, es un sistema de escritura logo-silábico. Cada signo puede tener varias posibilidades de lectura, correspondiente cada una de ellas, en ocasiones, a palabras relacionadas semánticamente; además, cada uno de estos valores puede teóricamente fonetizarse, es decir, convertirse en sílaba con valor exclusivamente fonético; y de cada una de estas sílabas, a su vez, pueden obtenerse valores secundarios, por ejemplo con sus correspondientes variantes sordas o sonoras.

Añadamos a ello que la escritura cuneiforme fue posteriormente utilizada por otros pueblos para reproducir su propia lengua, y nos encontraremos ante un complicado tipo de escritura en el que cada signo puede ser leído de varias formas diferentes. Estas múltiples posibilidades de lectura son, sin embargo, sólo teóricas, pues algunas de ellas se utilizaban sólo en unas épocas o escuelas y otras en momentos o lugares diferentes.

La asiriología ha conseguido desentrañar este sistema de una manera bastante satisfactoria, pero aún no ha llegado al punto de reconocer con precisión tradiciones escolares, ciudadanas o, simplemente, a establecer cánones paleográficos por épocas. De ello se derivan inconsistencias o ambigüedades en la lectura del signo que pueden llegar a comprometer seriamente la comprensión de algunos pasajes del texto.

Por otra parte, la escritura cuneiforme del Tercer Milenio a.C. no es un código que pretenda reproducir fielmente los sonidos de la lengua hablada. Hasta cierto punto puede hablarse de un «sistema nemotécnico» ${ }^{5}$ que evita muchos elementos gramaticales que a nosotros nos serían necesarios para reconocer el sentido de una oración o precisar el significado de una cadena verbal. Este es un rasgo característico, sobre todo, del Tercer Milenio a.C. Más tarde, cuando el sumerio deja de ser una lengua viva — pero que aún se escribe-, se irá afirmando la tendencia a reproducir en la escritura el mayor número posible de elementos gramaticales; es, en parte, este hecho el que nos permite conocer en profundidad la gramática sumeria, pero con las distorsiones que resultan del paso del tiempo y del tamiz acadio, un pueblo de habla semítica.

2) Nuestro conocimiento de la lengua sumeria, y en particular el de su léxico, tiene unas características también singulares. $\mathrm{Al}$ ser el sumerio una lengua sin "parientes» conocidos, su estudio pasa para nosotros por el que de él hicieron los acadios; éstos, considerando el sumerio

5 M. L. Thomsen, The Sumerian Language. An Introduction to its History and Grammatical Structure, Mesopotamia (Copenhagen Studies in Assyriology) 10, Copenhagen 1984, pp. 20-24. 
como lengua «clásica», confeccionaron «diccionarios» sumero-acadios de miles de palabras (listas léxicas).

A pesar de la riqueza de estas listas, no podemos dejar de tener la impresión de que nos hallamos ante un saber fosilizado, monolítico, que se va transmitiendo y aumentando durante generaciones como un gran «saco de ciencia» en el que cabe casi todo. Las distintas posibilidades de lectura de un signo, y los muchos significados de una misma palabra se yuxtaponen ante nosotros sin que se vislumbren más criterios que los didácticos, pues estas listas servían para la enseñanza de lo que los acadios concebían como el «sumerio clásico». No hay en ellas, por consiguiente, y salvo raras excepciones, criterios dialectales o de uso, y la separación del léxico o de determinadas expresiones según los géneros literarios se restringe a sólo un pequeño grupo de listas.

Es indudable, sin embargo, que a lo largo de sus más de mil años de historia conocida la lengua y el léxico sumerio evolucionaron, y que también había dialectos. Probablemente, muchas de las ambiguiedades y dificultades de este y otros textos derivan de los insuficientes avances de la asiriología en este terreno.

3) La época y la región a la que pertenece el texto tienen también su influencia en la comprensión del mismo. En nuestro caso, tenemos la fortuna de disponer de un corpus muy amplio de inscripciones reales conmemorativas o votivas de la llamada I Dinastía de Lagaš; en total tenemos más de seiscientos textos de este tipo, incluyendo duplicados o fragmentos ${ }^{6}$, lo que nos da muchas posibilidades de encontrar paralelos, variantes y contextos similares a un pasaje de interpretación problemática. Contamos, asimismo, con centenares de textos administrativos que permiten precisar y comprobar muchas de las cuestiones suscitadas en las inscripciones reales.

Así, por consiguiente, el caso de los textos de la I Dinastía de Lagaš es especialmente favorable, aunque, por ejemplo, no comparable al del período de la Tercera Dinastía de Ur, donde el número de textos publicados supera los treinta mil. Si a ello añadimos que, en el caso de los textos administrativos de Lagaš, nos encontramos frente a archivos muy específicos y homogéneos, no es raro que surjan en nuestro texto problemas de traducción o realidades sociales difíciles de comprender y de estudiar en un contexto más amplio y documentado.

Estas observaciones, si bien de manera superficial, ayudarán a entender el porqué de algunas dificultades de traducción en este y otros textos y las divergencias de interpretación --a menudo en pasajes cruciales - que existen entre los asiriólogos.

Este es el caso, por ejemplo, de los grupos sociales que se mencionan en nuestra inscripción. El problema aquí no es encontrar una traducción precisa del término: entendemos que esto es prácticamente imposible por la sencilla razón de que no existen entre nosotros realidades similares. Nuestro objetivo ha de ser, por el contrario, describir estos términos a través de su contenido, empleando para su estudio otros textos de los que podamos extraer datos relativos, por ejemplo, a las obligaciones laborales de estas personas, sus salarios, situación familiar, etc.

Este tipo de análisis, lamentablemente, no puede realizarse por el momento para los térmi-

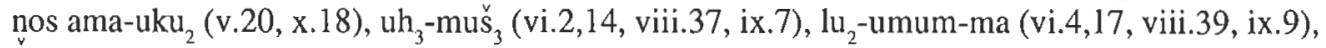
SUB-lugal (vi.37, x.20, xi.2,16) e igi-nu-du ${ }_{8}$ (vii.3,7, x.1). La comprensión de casi todos ellos es únicamente posible a través de la etimología o de contextos limitados, y los resultados son, por ahora, pobres y de escaso interés.

Véase un elenco en H. Steible, Die altsumerischen... I, pp. 35-44. 
En ocasiones ocurre también —como en todas las disciplinas-que el material sí podría ser lo suficientemente abundante como para realizar una investigación a fondo sobre determinados grupos de personas, aunque este estudio no se haya acometido todavía. Es el caso del papel de los «ancianos» en la sociedad mesopotámica (v. com. a ix.32-38).

El sentido de otros pasajes del texto, en cambio, puede entenderse a partir de contextos similares en otras tablillas administrativas: por ejemplo, la interpretación de iii.19-iv.8 se basa en otros documentos administrativos presargónicos de Lagaš, e incluso en la comparación con situaciones similares en el actual Iraq ${ }^{7}$.

Otras posibilidades de estudio y análisis nos las proporciona igualmente el riquísimo material epigráfico de Tell Mardikh-Ebla, cercano en el tiempo al de Lagaš, cuyos textos nos permiten precisar traducciones $(v i .25-31)^{8}$ que antes tenían bases menos sólidas.

Otros problemas de traducción derivan de prejuicios por parte de algunos autores, quienes a veces parten de alguna idea previa para interpretar el texto a su gusto. Este es el caso, como ha mostrado Foster ${ }^{9}$, del pasaje iii.15-18, para el que nos encontramos las siguientes traducciones:

- A. Deimel: «Die šutug-Priester mussten den Pachtzins (vor dem Magazinaufseher) im Marschlande abmessen ${ }^{10}$.

- S.N. Kramer: «The barley rations of the guda used to be measured out (to their disadvantage) in the Ashte»".

- M. Lambert: «Les shutug faisaient mesurer les impositions d'orge dans le (quartier du) Marais»'12.

La versión de Kramer, sin duda la más llamativa, implica que los sacerdotes-gudug eran maltratados. Sin embargo, no hay nada en el texto que apoye la interpretación de «en perjuicio suyo» para el verbo $\mathrm{ag}_{2}$.

El progreso en la investigación lexicográfica, finalmente, está contribuyendo en no poco al esclarecimiento de pasajes cuya interpretación no era demasiado satisfactoria. De esta manera, el análisis de P. Steinkeller ${ }^{13}$ propuesto para la palabra $e_{2}-\mathrm{ES}_{2}$ («prisión») ha permitido reconsiderar la interpretación (también desde el punto de vista sintáctico y morfológico) de ix.20-29 y entenderlo como una proclamación de amnistía para prisioneros por deudas, estafadores, ladrones y asesinos.

\section{LAS «REFORMAS»}

El texto de Las «Reformas» de UruKAgina cuenta con cuatro partes. La primera (i.1-iii.2) es una introducción con una dedicatoria al dios Ningirsu y una breve descripción de las actividades

7 Cf. P. Steinkeller, «The Renting of Fields in Early Mesopotamia and the Development of the Concept of 'Interest' in Sumerian», Journal of the Economic and Social History of the Orient 24 (1981) 132-133; D. A. Foxvog, "A New Lagaš Text bearing on Uruinimgina's Reforms", Joumal of Cuneiform Studies 46 (1994) 11-15.

8 Cf. F. Pomponio, «Urukagina 4 VII 11 and an Administrative Term from the Ebla Texts», Journal of Cuneiform Studies 36 (1984) 96-100.

9 B. Foster, «A New Look at the Sumerian Temple State», Journal of the Economic and Social History of the Orient 24 (1981) 234.

10 A. Deimel, «Die Reformtexte Urukaginas», Orientalia SP 2 (1920) 7.

11 S.N. Kramer, The Sumerians, Their History, Culture and Character, Chicago 1963, p. 320.

12 M. Lambert, «Les 'reformes' d'Urukagina», Revue d'Assyriologie 50 (1956) 171.

13 P. Steinkeller, «The Reforms of UruKAgina and an Early Sumerian Term for 'Prison'», Aula Orientalis 9 $(1991)=F s$. M. Civil, pp. 227-233. 
del rey como constructor. La segunda (iii.3-vii.11) contiene una lista de antiguas prácticas que se consideran «abusos de poder» cometidos en su mayoría por la familia real o por sus funcionarios sobre la población o los sacerdotes. La tercera parte (vii.12-xi.19) describe las soluciones a estos «abusos» propuestas por UruKAgina una vez que éste ha sido elegido como rey. El texto concluye con una cuarta sección (xi.20-xii.21) donde el monarca anuncia la liberación de ciudadanos encarcelados y la protección de viudas y huérfanos, y describe a continuación la construcción de un canal.

Una de las novedades estructurales de este texto es la correspondencia casi exacta que muestra entre los «abusos» descritos en la segunda parte y las «reformas» propuestas en la tercera ${ }^{14}$ :

\section{«Abusos»}

$\S$ la Irregularidades cometidas por supervisores del transporte en barca, del ganado y de la pesca (iii.6-14) ${ }^{15}$

$\S 2$ a Irregularidades cometidas por el supervisor de los silos sobre las contribuciones de cebada de los sacerdotes-gudug (iii. 15-18) $)^{16}$

\section{§ 3a Irregularidades cometidas por el inspec- tor de tasas pagadas por el ganado naci- do en campos de alquiler (iii.19-iv.8) ${ }^{17}$}

§ 4a Utilización indebida de las propiedades de los templos por parte de la familia real (iv. $9-25)^{18}$

\section{«Reformas»}

$=\S 1 \mathrm{~b}$ Eliminación de estas irregularidades (vii.27-viii.1)

$=\S 2 \mathrm{~b} \quad$ Eliminación de estas irregularidades (viii.2-5)

$=\S 3 \mathrm{~b}$ Eliminación de estas irregularidades (viii.7-10)

$=\S 4 \mathrm{~b}$ Concesion a los dioses del control sobre las propiedades de la familia real (viii.16-27) ${ }^{19}$

14 Véase a este respecto: M. Lambert, «Les 'reformes' d'Urukagina», Revue d'Assyriologie 50 (1956) 169-184; I.M. Diakonoff, «Some Remarks on the 'Reforms' of Urukagina», Revue d'Assyriologie 52 (1958) 1-15; B. Hruska, «Die innere Struktur der Reformtexte Urukaginas von Lagaš», Archiv Orientalni 41 (1973) 4-13 y $104-132$.

15 Ukg 5:iii,6-14 = Ukg 1:iv,10-17 (= Ukg 2:iii', l'-3').

16 Ukg 5:iii.15-17 = Ukg 1:iv.18-20 (= Ukg 2:iii'.4'-6').

Ukg 5:iii.15-17 = Ukg 6:i.5'-9'. En la versión de Ukg 6 se añade: gudu -be $_{2}$-ne / e e $_{2}$-se-gub-ba-bi Ambar ${ }^{\text {'ki }}$ a $\mathrm{i}_{3}-\mathrm{du} \mathrm{u}_{3}-\mathrm{du_{3 }}$ “Los sacerdotes-gudug tenían que construir un almacén en Ambar para esas contribuciones de cebada».

17 Ukg 5:iv. 3-8 = Ukg 1:iv.26-31.

Ukg 5:iv,3-8 $\approx$ Ukg 6:i.11'-21'. La versión de este «abuso» en Ukg 6 es la siguiente: [agrig-ge-n]e / ugula-ne / gala-e-ne / engar-re ${ }_{2}$-ne / lu -bappir $_{3}-k_{4}$-ne / udu-siki $u_{3}-$ mu-DU / $e_{2}$-gal-la $u_{3}-u_{4} / u_{4}$-da udu e-had $/$ siki-bi e $_{2}$-gal-la a-ba-DU / $\mathrm{ku}_{3}$ gin $_{2}-5$-am 6 / e-ga - ga $_{2}$-ne «Si los administradores, supervisores, cantantes del culto, los labradores, (o) los maestros cerveceros traían una oveja de lana y la esquilaban en el palacio, si la oveja era pura y la lana se entregaba en el palacio, también tenían que pagar cinco siclos de plata».

18 Ukg 5:iv.9-13= Ukg 6:i.22'-26'.

19 Ukg 5:viii.16-27 = Ukg 1:v.1"-10". En la versión de Ukg 1, la fraseología utilizada es, sin embargo,

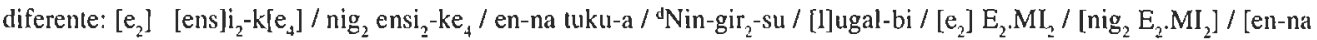
tuku-a] / [ $\left.{ }^{\mathrm{B}} \mathrm{Ba}-\mathrm{ba}_{6}\right] /$ [nin-bi] / [...] «[él estableci6?] a Ningirsu como señor de la hacienda del ensi ${ }_{2}$, de las propiedades del ensi, de tanto como él poseía; a Baba como señora de la hacienda de la 'Casa de la Mujer', de las propiedades de la 'Casa de la Mujer', de tanto como ella poseía [...]». 
$\S 5 \mathrm{a}$ Irregularidades cometidas por el inspec- $=\S 5 \mathrm{~b}$ tor de las contribuciones entregadas por los administradores del templo (v.1-18)

$\S 6 \mathrm{a}$ Costumbre que permite a los adminis- $=\S 6 \mathrm{~b}$ Abolición de esta costumbre (x.17tradores-GAR del templo apropiarse de los productos de los huertos de los ama$\mathrm{uku}_{2}$ (v.19-23)

$\S 7 \mathrm{a}$ Irregularidades cometidas en el pago por $=\S 7 \mathrm{~b}$ ritos funerarios (v.24-vi.18)

$\S 8 \mathrm{a}$ Irregularidades cometidas por los arte- $=\S 8 \mathrm{~b}$ sanos (vi.19-21)

$\S 9 \mathrm{a}$ Irregularidades cometidas por «parejas $=\S 9 \mathrm{~b}$ de obreros» (vi.19-21)

$\S 10 \mathrm{a}$ Irregularidades cometidas en las propie- $=$ dades de la familia real (vi.25-31)

§ 11a Descripción del radio de acción de los inspectores reales (vi.32-36)

$=\S 11 \mathrm{~b}$ Supresión de estos inspectores (viii.28-31)

$\S 12 \mathrm{a}$ Abusos cometidos por el ŠUB-lugal sobre el igi-nu-du ${ }_{8}(\text { vi.37-vii.8) })^{22}$
Eliminación de estas irregularidades (viii.11-15)
Eliminación de estas irregularidades y regulación de los pagos por ritos funerarios y ceremonias de lamentación (viii.32-x.10) ${ }^{21}$

Eliminación de estas irregularidades (x.14-16)

Eliminación de estas irregularidades (x.11-13)

cf. $\S 4 b$

§ 13b Prohibición de abusos de un «hombre grande» sobre las propiedades de un ŚUB-lugal (x.20-xi.18)

20 Ukg 5:x.17-19= Ukg l:v.l'-5' (esta versión añade la prohibición de recoger los frutos mencionados en Ukg 5:v.22-23).

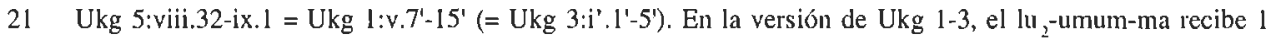
gur $^{\text {sic!) }}$ de cebada.

Ukg 5:ix.15-2l = Ukg 1:vi.3'-9' (= Ukg 3:ii'. l'-6'). Ukg 1:vi.3' anota 480 en lugar de 420.

Ukg 5:ix.32-38 = Ukg 1:vi.20'-25'.

Ukg 5:x.5-6 = Ukg l:vi. $1^{\prime}-2$ '.

Ukg 5:x.11 = Ukg 1:vi.26'.

22 Ukg 5:vi.37-vii.8 = Ukg 6:ii.0'-9'. La versión de este «abuso» en Ukg 6 es la siguiente: [a-muš-du]/

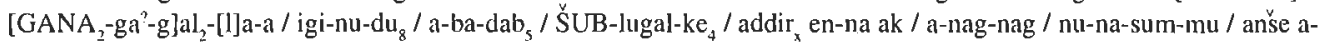
nag-nag / nu-ba-sum-mu "Si se cogía a un igi-nu-du ${ }_{8}$ para (trabajar en) los canales de irrigación que estaban en el

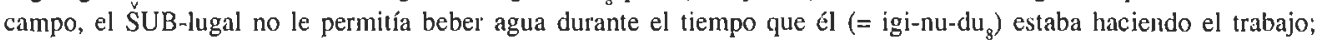
tampoco le daba agua para beber al asno». 
A los «abusos» y «leformas» aquí descritos habría que añadir otros no mencionados en las versiones I (Ukg 1-3,62) y II (Ukg 4-5, 60) y que sí aparecen en la versión III (Ukg 6). Este último texto, de difícil clasificación, contiene una sección en la que se describe el conflicto fronterizo entre Lagaš y Umma, y otra en la que se enumeran los «abusos» y las «reformas» promulgadas por UruKAgina, Las novedades de esta sección con respecto a las versiones I y II son las siguientes:

\section{«Abusos»:}

[...] / $\mathrm{ku}_{3}{ }^{\mathrm{r}} \mathrm{u}_{3}{ }^{\top}$-ri-ri / udu u $\mathrm{u}_{3}-\mathrm{sa}_{10} / \mathrm{udu}$-ba udu ša ${ }_{6}$-ga-bi / lu $\mathrm{u}_{2}$ ba-ta-tum - -mu «[...] acumulaba plata (y) compraba ovejas, (entonces) se llevaba las ovejas de buena calidad entre esas ovejas» (Ukg 6:i.0'-4').

dumu-uku ${ }_{2}-\mathrm{ra}_{2}-\mathrm{ke}_{4} /$ HAR.SAGxHA-na / $\mathrm{u}_{3}$-mu-ak / $\mathrm{ku}_{6}-\mathrm{bi} \mathrm{lu_{2 }}$ ba-da $-\mathrm{kar}-\mathrm{re}_{2} / \mathrm{lu} \mathrm{u}_{2}-\mathrm{bi}_{3}-\mathrm{d} \mathrm{Utu} \mathrm{i}_{3}-\mathrm{e}$ «cuando un dumu-uku ${ }_{2}$ hacía un 'agujero para peces', se llevaban esos peces, (y) ese hombre (= dumu-uku ${ }_{2}$ (sólo) podía decir '¡Oh, Utu!'» (Ukg 6:ii.10'-14').

$\mathrm{lu}_{2}$ dam $\mathrm{u}_{3}-\mathrm{tag}_{4} / \mathrm{ku}_{3}$ gin $_{2}-5-\mathrm{am}_{6} / \mathrm{ensi}_{2}-\mathrm{ke}_{4} / \mathrm{ba}-\mathrm{DU} / \mathrm{ku}_{3} \mathrm{gin}_{2}-1-\mathrm{am}_{6} /$ sukkal-mah-e / ba-DU "Cuando un hombre repudiaba a su esposa, el ensi ${ }_{2}$ se llevaba cinco siclos de plata, (y) el gran visir se llevaba un siclo de plata» (Ukg 6:ii.15'-21').

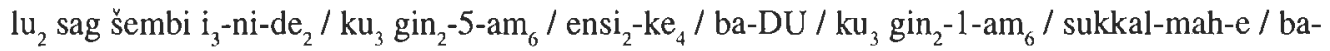
DU / $\mathrm{ku}_{3}$ gin $_{2}-1-\mathrm{am}_{6}$ abgal $_{x}-\mathrm{le}$ ba-DU «Cuando un hombre vertía antimonio sobre la cabeza (de otro), el ensi ${ }_{2}$ se llevaba cinco siclos de plata, el gran visir se llevaba un siclo de plata (y) el 'sabio' se llevaba un siclo de plata» (Ukg 6:ii.22'-31').

$\mathrm{lu}_{2} \mathrm{GIR}_{3}$-a DU / TUG $\mathrm{GA}^{\prime} \mathrm{x}^{\top}[\ldots] /[\ldots]$ «Cuando un hombre ... [...]» (Ukg 6:ii. 32'-...).

\section{«Reformas»:}

${ }^{\top} \mathrm{ku}_{3} \mathrm{x}^{\top}[\ldots] /$ ensi $_{2}-\mathrm{ke}_{4} /$ sukkal-le / abgal $-\mathrm{le} / \mathrm{nu}-\mathrm{ba}-\mathrm{tum}_{3}$ «... de plata el ensi, ${ }_{2}$, el visir y el 'sabio' no se llevarán» (Ukg 6:iii.1'-5').

dumu-uku ${ }_{2}-\mathrm{ra}_{2}-\mathrm{ke}_{4} / \mathrm{HAR} . S A G \times H A-n a / \mathrm{u}_{3}$-ak / $\mathrm{ku}_{6}$-bi lu $\mathrm{u}_{2}$ nu-ba-da $-\mathrm{kar}-\mathrm{re}_{2}$ «Cuando un dumu$\mathrm{uku}_{2}$ haga un 'agujero para peces', nadie se llevará esos peces» (Ukg 6:iii.6'-9').

nig ${ }_{2}-z u h-a / z a-a s_{2}-d a-b i i_{3}-s u b / n i g_{2}-u_{2}-g_{x}-d e_{2}-a / a-b u l_{x}-l a ~ e-l a_{2} \ll E l$ crimen del robo se ha eliminado, (y) los objetos perdidos se cuelgan (ahora) en la puerta de la ciudad» (Ukg 6:ili.10'-13').

munus-e nita-ra / $\mathrm{r}^{\mathrm{X}} \times \mathrm{x}^{\top} \mathrm{DAG}$-ba $\mathrm{i}_{3}$-ni-du ${ }_{11} /$ munus-ba ka-ka-ni / BAHAR $\mathrm{i}_{3}$-šuš / BAHAR 2 -bi / a-bul-la e-la ${ }_{x}$ «Cuando una mujer contra un hombre diga: '.. ', la boca de esa mujer será aplastada con un ladrillo cocido, (y) ese ladrillo cocido se colgará en la puerta de la ciudad» (Ukg 6:iii.14'-19').

munus ud-bi-ta-ke $e_{4}$-ne / nita-2-ta / $i_{3}$-tuku-am $/$ munus ud-da-e-ne / za-aš 2 -da-bi $i_{3}$-šub «Las mujeres de antes tenían dos hombres, (pero) las mujeres de hoy evitan este crimen» (Ukg 6:iii.20'-24'). [La palabra za-aš ${ }_{2}$-da podría interpretarse también como «esclavitud por deudas», 
en lugar de «crimen»; en este caso, se podría estar también haciendo referencia a las obligaciones hacia dos hombres que tendría una mujer esclavizada por deudas.]

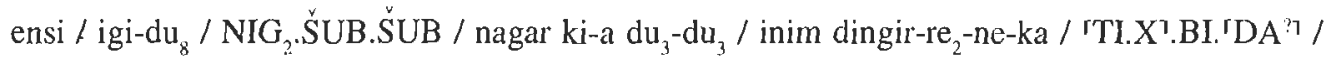
[...] «El intérprete de sueños, el vidente, el ..., el carpintero que construye en un lugar, en la palabra de los dioses ... [...]» (Ukg 6:iii.25'-...).

\section{LA CUESTIÓN DE LA «CIUDAD-TEMPIO» SUMERIA}

De todas estas «reformas», la más conocida es, sin duda, aquella que concede a los dioses las propiedades que antes estaban bajo el control de la familia real ( $\$ 4$ b).

Este pasaje ha sido interpretado en el sentido de que las unidades económicas y campos en cuestión habían pertenecido anteriormente a los dioses; dichas propiedades fueron después usurpadas por el rey y su familia, y ahora, gracias a UruKAgina, se devolvían a sus dueños originales. Las bases para dicha reconstrucción se encontraban en la propia inscripción de Las "Reformas» de UruKAgina y en un grupo de textos administrativos procedentes de Lagaš (c. 1700 tablillas).

El impulsor de esta teoría fue Deimel ${ }^{23}$ — después seguido por Schneider ${ }^{24}$-, quien reconoció en la documentación administrativa un complejo económico que, bajo los reinados de Enéntarzi y Lugalanda (predecesores de UruKAgina), era denominado $\mathrm{E}_{2} \cdot \mathrm{MI}_{2}$ (lit. «Casa de la Mujer»); éste estuvo administrado por Dimtur y Baragnamtara, esposas, respectivamente, de Enentarzi y Lugalanda. Con UruKAgina, sin embargo, el $\mathrm{E}_{2} \cdot \mathrm{MI}_{2}$ habría pasado a denominarse $\mathrm{e}_{2}{ }^{-}{ }^{\mathrm{d}} \mathrm{Ba}-\mathrm{ba}_{6}$ ( Casa/Templo de la diosa Baba») ${ }^{25}$; ello mostraría la eficacia de las reformas de este monarca al imponer a los dioses como propietarios de la hacienda real. Además, a la vista de la importancia económica del $\mathrm{e}_{2}{ }^{-}{ }^{\mathrm{B}} \mathrm{Ba}-\mathrm{ba}_{6} \mathrm{y}$ de la información contenida en los textos administrativos sobre otros templos y santuarios, se pensó que, inicialmente, las tierras y gentes de la ciudad pertenecían a los templos. En este contexto, el gobernante (ensi $i_{2}$ y su familia eran considerados meros representantes terrenos de la familia divina, y la figura de UruKAgina habría surgido para corregir la subversión de este principio llevada a cabo por sus antecesores.

A partir de estos estudios se fue consolidando el concepto de ciudad-templo, convenientemente formulado después por A. Falkenstein ${ }^{26}$ y aceptado por muchos otros autores. Sin embargo, nuevos análisis realizados por $\mathrm{K}$. Maekawa ${ }^{27}$ sobre los textos administrativos de Lagaš han permitido reconsiderar el papel del palacio en época presargónica y han mostrado que:

23 A. Deimel, Die sumerische Tempelstadt, Essen 1920; id., Sumerische Tempelwirtschaft zur Zeit Urukaginas und seiner Vorgänger, Analecta Orientalia 2, Roma 1931. Véase también N. Schneider, Die sumerische Tempelstad, Essen 1920 .

24 N. Schneider, Die sumerische ...

25 Tal afirmación puede verificarse también a través de otros datos: por ejemplo, el intendente Eniggal se hará un nuevo sello en el que su título de «escriba de la Casa de la Mujer» (En-ig-gal / dub-sar $\mathrm{E}_{2}, \mathrm{MI}_{2}$ [Lug 6-11]) es reemplazado por' el de «(escriba) de la Casa de Baba» (En-ig-gal / dub-sar 'Ba-ba ${ }_{6}$ [Lug 12]).

26 A. Falkenstein, "La Cité-Temple Sumérienne», Cahiers de l'Hisioire Mondiale 1 (1954) 784-814. Traducción al inglés, por M. de J. Ellis, en Monographs on the Ancient Near East 1/1, Malibu 1974.

27 K. Maekawa, "The Development of the É.Mí in Lagaš during Early Dynastic III», Mesopotamia 8-9 (197374) 77-144. Opiniones contrarias a las del concepto clásico de ciudad-templo han sido también expresadas, por ejemplo, por B.R. Foster, "A New Look ...", pp. 235-24l; H.J. Nissen, The Early History of the Ancient Near East. $9000-2000$ B.C., Chicago 1988 [Darmstadt 1983], p. 148; G. Pettinato, I Sumeri, Milán 1992, p. 234. 
1) El papel del palacio en la vida económica, social y política de la ciudad-estado sumeria es ya muy significativo desde la época de los textos arcaicos de Uruk, Ur y Jamdat-Nașr ${ }^{28}$. Más tarde, en las inscripciones reales de los primeros reyes de la I Dinastía de Lagaš, encontramos formulado el concepto de la realeza como institución vinculada a la voluntad divina. Dicho concepto se hallará por vez primera claramente atestiguado en los textos administrativos de la época de UruKAgina. En este contexto, el control del templo del dios Ningirsu por parte del palacio existiría ya con los predecesores de UruKAgina y continuó durante su reinado.

2) La expansión del $\mathrm{E}_{2} \cdot \mathrm{MI}_{2}$ ( «Casa de la Mujer»), como organización estatal básica de la ciudad de Lagas ${ }^{29}$ en época de Enentarzi y Lugalanda, contribuyó al desarrollo de la teoría que concebía a la familia real como administradora terrena de las propiedades de la familia divina ${ }^{30}$. UruKAgina habría sido el responsable de proclamar oficalmente esta concepción del poder.

3) En consecuencia, UruKAgina creó una nueva unidad económica bajo el patrocinio del dios Igalim (hijo de Ningirsu), pero esta organización no prosperó y se disolvió durante el segundo o tercer año de su mandato (= Ul 162 ). Tras este fracaso, y durante su tercer año de gobierno (= Ul 2), UruKAgina retomó el modelo administrativo de su antecesor Lugalanda. Así, las propiedades de sus hijos se subordinaron al $\mathrm{E}_{2} \cdot \mathrm{MI}_{2}$, que ahora pasó a llamarse $\mathrm{e}_{2}{ }^{\mathrm{d}} \mathrm{Ba}^{-} \mathrm{ba}{ }_{6}$ («Casa de la diosa Baba»), de hecho administrado, sin embargo, por su esposa Šaša ${ }^{31}$.

Por lo tanto, y según se desprende de la documentación administrativa, las «reformas» de UruKAgina no supusieron ninguna devolución de propiedades a los templos ${ }^{32}$. Las modificaciones en este terreno se redujeron, esencialmente, a un cambio en el nombre de la organización económica administrada por la esposa del rey; es cierto que dicha organización adquirió una mayor importancia económica a partir del tercer año de gobierno de UruKAgina (=U1 2), pero no se observa ningún cambio significativo en su estructura básica. UruKAgina creó, asimismo, una nueva organización económica nominalmente tutelada por Igalim, hijo del dios Ningirsu, pero su vida fue efímera. Así pues, ambas organizaciones - de acuerdo con una ideología que venía gestándose desde hacía varias generaciones- pasaron a estar nominalmente bajo el patrocinio de los miembros de la familia divina (una de ellas durante un corto período de tiempo), pero, de hecho, siguieron estando controladas, como antes, por la familia real.

28 Sobre la identificación de los edificios públicos en los niveles más antiguos de estas ciudades, v, también H.J. Nissen, The Early History ..., pp. 96-98, 140; G. Pettinato, I Sumeri, pp. 117 ss., 192 ss., 254-255.

29 Sobre esta importante característica del $\mathrm{E}_{2}, \mathrm{MI}_{2}$, v. J.M. Asher-Greve, Frauen in altsumerischer Zeit, Bibliotheca Mesopotamica 18, Malibu 1985, p. 150. Sobre el tipo de personas vinculadas a esta clase de organizaciones económicas, v. I.J. Gelb, «Household and Family in Early Mesopotamia», en E. Lipinski (ed.), State and Temple Economy in the Ancient Near East 1, Orientalia Lovaniensia Analecta 5, Leuven 1979, pp. 23-24.

30 A ello habría contribuido también la existencia de propiedades y personal dependientes de los hijos del ensi ${ }_{2}$. Este hecho, que no se halla documentado para Geme-Baba, la hija de Enentarzi y Dimtur, sí lo está para $\mathrm{MI}_{2}$-šaga y Geme-Nanše, hijas de Lugalanda y Baragnamtara: v. J.M. Asher-Greve, Frauen ..., pp. 151-152.

31 Hay que notar, además, que las propiedades personales $\left(\mathrm{u}_{2}\right.$-rum) de Dimtur, Baragnamtara y Lugalanda estaban bien diferenciadas del $\mathrm{E}_{2}, \mathrm{ML}_{2}$, y que UruKAgina no las confiscó para administrarlas a través del $\mathrm{E}_{2}, \mathrm{MI}_{2}$. Muy al contrario, la propiedad personal ( $\mathrm{u}_{2}$-rum) de Sasa iba a estar administrada ahora desde el palacio (e $\mathrm{e}_{2}$-gal): v. J.M. AsherGreve, Frauen ..., p. 154.

32 Cf. también B. Foster, «A New Look ...», pp. 237-241. 


\section{LA SUCESIÓN DINÁSTICA EN LAGAS̆}

A pesar del continuismo administrativo en el que quedaron las «reformas» de UruKAgina, llama la atención el hincapié que se hace en el texto en responsabilizar a las anteriores familias gobernantes de abusos realizados contra la clase sacerdotal y la población en general. En efecto (vid. supra), de los trece «abusos» o «reformas» descritos en el texto, en casi todos los casos se ven incriminados, por distintas causas, la familia real, o funcionarios o dependientes suyos, por irregularidades o abusos cometidos contra las propiedades de los templos, los intereses de los sacerdotes o las capas más desfavorecidas de la población.

Las razones de esta actitud pueden buscarse, en primera instancia, en la condición de UruKAgina como rey no legítimo según los cauces habituales de sucesión dinástica. Las circunstancias de su llegada al poder y sus precedentes se nos revelan en los textos a través de los siguientes datos:

Tras Enanatum II (perteneciente a la dinastía inaugurada por Ur-Nanše), a raíz de las luchas contra los elamitas y los enfrentamientos con la ciudad de Umma por la llanura del Guedena, Lagaš va a entrar en un período de crisis dinástica. Su sucesor, Enentarzi, con quien no le unían vínculos de sangre, había sido previamente, durante varios años, el administrador principal (sanga) del templo de Ningirsu. La relativa independencia social y política del cargo de sanga, constatable a partir de diversos datos, estuvo seguramente relacionada en este tiempo, según ha hecho notar Maekawa ${ }^{33}$, con el mencionado debilitamiento del poder real, y pudo propiciar la llegada al trono de Enentarzi. A éste le sucedería después su hijo Lugalanda, con quien, como más adelante veremos, UruKAgina iba a mantener una relación bastante peculiar.

Lugalanda fue sustituido por U1uKAgina en su séptimo año de reinado ${ }^{34}$. Urukagina, hijo de Urutu $^{35}$, no pertenecía a la línea dinástica de Enentarzi, pero no parece que su llegada al poder hubiese estado marcada por hechos violentos (vid. infra).

33 K. Maekawa, «The Development ...», pp. 137-138.

34 Las dataciones de los textos administrativos atestiguan el ascenso al poder de UruKAgina como ensi i $_{2}$ de Lagaš al menos en el tercer mes del séptimo y último año de reinado de Lugalanda: así, F. Thureau-Dangin, Recueil de tablettes chaldéennes, París 1903, $\mathrm{n}^{\circ} 55$, por ej., es un texto datado en el primer mes del año 7 de Lugalanda, mientras que F. Allotte de la Fuÿe, Documents présargoniques, París 1908-1920, n 152, perteneciente al tercer mes del mismo año, es registrado ya como año de UruKAgina ensi ${ }_{2}$ de Lagaš.

35 Cf. T. Kobayashi, «The ki-a-nag of Enentarzi», Orient 21 (1985) 12. 


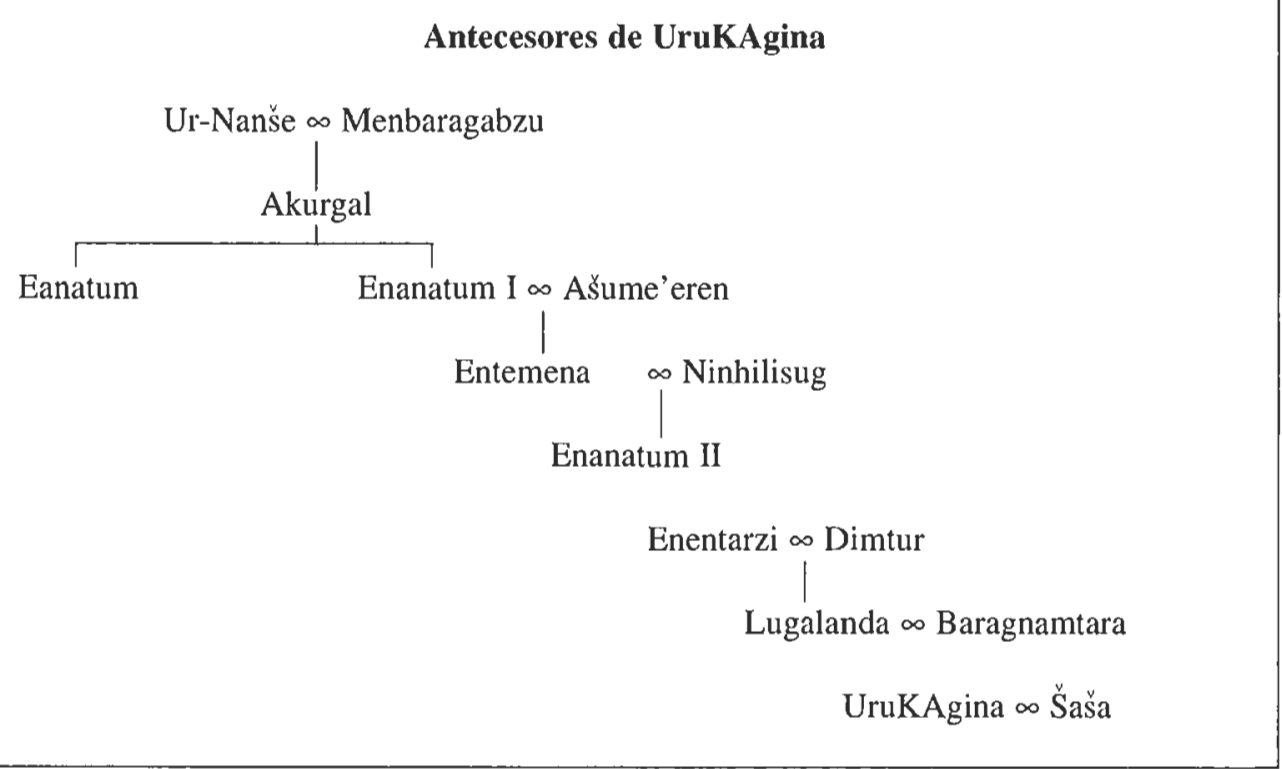

\section{LA TITULATURA}

Durante el primer año de su mandato, UruKAgina, al igual que sus antecesores, ostenta el título de ensi. En el segundo año, sin embargo, asume la nam-lugal («realeza»), y pasa a denominarse lugal («rey»); a partir de este momento comenzará una nueva era en Lagaš, pues los textos adoptan un sistema de datación que arranca desde el primer año de UruKAgina como lugal, borrando de la memoria del calendario el primer año del rey como ensi. ${ }_{2}$. No están todavía demasiado claras las diferencias entre uno y otro título en época presargónica ${ }^{36}$, y particular-

36 Una buena síntesis sobre la titulatura real en época presargónica se encontrará en W.W. Hallo, Early Mesopotamian Royal Tilles: A Philologic and Historical Analysis, American Oriental Series 43, New Haven 1957, pp. 3-48. En este trabajo se muestra cómo el título de lugal aparece inicialmente ligado a la ciudad de Ur (textos arcaicos de $U_{1}$ ), mientras que el título de en aparecería vinculado a Uruk desde tiempos aún más antiguos (Uruk IV); el término ensi $_{2}$ (PA.TE.SI) se encuentra, por su parte, registrado ya en los textos arcaicos de Ur y en los de Fara (NIG .PA.TE.SI), pero, en opinión de Hallo, no sc utilizará para designar a un soberano independiente hasta la época de Eanatum de Lagaš.

Las connotaciones específicas de uno u otro título dependerían - según apuntó Th. Jacobsen, «Early Political Development in Mesopotamia», Zeitschrift für Assyriologie 18 (1957) 91-140, y después olros autores- de la época y el lugar en que fuesen aplicados; así, con el término en nos encontraríamos ante la máxima expresión de un poder que inicialmente, en época de Uruk, era de carácter religioso; más tarde, la generalización de los conflictos entre las ciudades-estado habrían propiciado la aparición de un poder civil alternativo, el lugal, quien habría terminado por convertirse en el soberano absoluto del Estado. En este contexto, el ensi, se entendía como un cargo con un poder territorial de dimensiones más reducidas que el del lugal.

Recientemente, M. Liverani (Antico Oriente. Storia, società, economia, Roma-Bari 1988, pp. 183-184) ha intentado matizar esta cuestión: según este autor, en época presargónica, con el título de en se recordaba el papel del templo como lugar de origen del poder real, con el de ensi, se estaría subrayando la función del dinasta como administrador de 
mente en Lagaš la situación se nos presenta bastante confusa. En efecto, en esta ciudad UruKAgina no fue el único rey que utilizó tanto el título de ensi ${ }_{2}$ como el de lugal; como a continuación se detalla, también algunos de sus predecesores utilizaron ambos títulos en sus inscripciones:

- Ur-Nanše: se denomina a sí mismo lugal de Lagaš en todas sus inscripciones, excepto en Urn 50; a él se refieren también, como lugal de Lagaš, Akurgal (Akg 1), Eanatum (Ean 1) y Entemena (Ent 1, 8). Ur-Nanše, además, se denomina a sí mismo ensi, de Lagaš en Urn 50, y es denominado como tal por Eanatum (Ean 2,3).

- Akurgal: es denominado lugal de Lagaš por Eanatum (Ean 1). Akurgal, además, se denomina a sí mismo ensi ${ }_{2}$ de Lagaš en las dos únicas inscripciones que se le conocen (Akg 1, 7), y es denominado como tal por Eanatum (Ean 2, 3, 7, 11, 22, 60, 69) y Enanatum I (En I 2, 9, 18, 20, 26, 28, 29, 33, 35).

- Eanatum: Eanatum se denomina a sí mismo lugal de Lagaš en dos inscripciones (Ean 1, 64), en una de las cuales (Ean 1) alude a la concesión de la realeza de Lagaš (nam-lugal

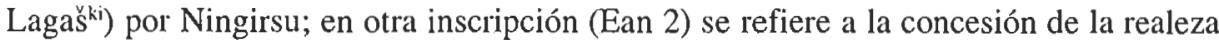
de Kiš (nam-lugal Kiš ${ }^{k i}$ ) por Inanna. En el resto de las inscripciones, Eanatum se denomina a sí mismo y es denominado ensi ${ }_{2}$ de Lagaš.

las propiedades del dios de la ciudad, y con el de lugal se haría más hincapié en sus cualidades humanas (en sentido físico y socio-económico); Liverani anota, adcmás, que el término ensi, podía implicar una dependencia también a nivel humano, por lo que los reyes más poderosos, cuando desarrollaban una política hegemónica respecto a otras ciudadesestado, tendían a adoptar el título de lugaí.

La mayor oposición a esta reconstrucción de la evolución del poder político en Mesopotamia desde la época de Uruk hasta la Dinastía de Akkad ha provenido, sin embargo, de H.J. Nissen (The Early History ..., pp. 140-144). Este autor ha puesto de relieve, con contundentes razones, la inexistencia de una contraposición progresiva entre poder real (teóricamente representado por el lugal) y poder religioso (teóricamente representado por el en), y la inconveniencia de extrapolar datos, indiscriminadamente, de uno a otto período o área geográfica. Así, según ha mostrado Nissen, una lista de profesiones del período de Jamder Nasr sitúa al en y el lugal en posiciones no relevantes, por lo que probablemente cstos tílulos no tenían nada que ver en esta época con el significado que posteriornente adquirieron; y cuando ambos toman el significado conocido en el Dinástico Antiguo III, no lo hacen partiendo de un contraste entre «sumo sacerdote» y «rey», sino que dependen de diferentes tradiciones en las que las funciones de lugal y en no habrían sido muy distintas.

G. Pettinato (I Sumeri, pp. 238-256), por su parte, ha propuesto entender la figura del en en época de Uruk como la máxima expresión de un poder dc ámbito supranacional (concepto que se habría traspasado a Ebla). La figura del lugal como soberano absoluto sería característica de la tradición de Kiš, y habría terminado por imponerse, como modelo de poder político, tanto a la del en, como a la del ensi, (característica de Lagaš y de otras ciudades).

Th. Jacobsen («The Term Ensi», Aula Orientalis 9 [1991] = Fs. M. Civil, pp. 113-12I) ha estudiado el término ensi, con criterios esencialmente filológicos, concluyendo lo siguiente (p. 120): «to sum up we would thus argue that the original meaning of the term ensît $(k)$ is 'efficient manager of donkeys'. That this implied organization of the yearly ploughing of temple or town, and that the broad authority this involved made the step to city ruler an easy and common one, thereby giving rise to a secondary meaning 'city ruler'. Akkadian and Sumerian employed the word in both meanings, depending on context to keep them distinct».

Finalmente, W. Heimpel («Herrentum und Königtum im vor- und frühgeschichtlichen Alten Orient», Zeitschrift fiir Assyriologie 82 [1992] 4-21) ha señalado que el cargo de en («das Herrentum») fue la forma de poder característica de la Uruk arcaica, desde donde se extendió a otras zonas del Próximo Oriente, hasta que fue reemplazada, en el Dinástico Antiguo, por el poder del lugal («das Königtum»); la diferencia esencial entre ambos poderes —además de otras apuntadas por este autor - estribaría en que, al contrario que el cargo de en, el de lugal se regía por los principios de sucesión dinástica. 
- Enanatum I: es denominado ensi ${ }_{2}$ de Lagaš en todas las inscripciones; en dos de ellas (En I 9,33), sin embargo, alude a la concesión de la realeza de Lagaš (nam-lugal Lagaški) por el dios Lugal-URUxGANA ${ }_{2}$-tenû $\hat{u}^{37}$.

- Entemena: es denominado ensi ${ }_{2}$ de Lagaš en todas las inscripciones; en una de ellas (Ent 26), sin embargo, alude a la concesión de la realeza de Lagaš (nam-lugal Lagaški) por Nanše.

- Enanatum II: es denominado ensi, en todas las inscripciones.

- Enentarzi: es denominado ensi, en todas las inscripciones (textos administrativos).

- Lugalanda: es denominado ensi ${ }_{2}$ en todas las inscripciones (textos administrativos).

- UruKAgina: es denominado ensi ${ }_{2}$ de Lagaš (textos administrativos) durante su primer año de reinado. A partir del segundo año adopta el título de lugal; como tal, recibe la realeza de Lagaš (nam-lugal Lagaš ${ }^{k i}$ ) por medio de Ningirsu (Ukg 4-5) y la realeza de Girsu (Ukg 1).

La misma ambigüedad puede observarse también, en ocasiones, en otros contextos de la documentación administrativa: así, por ejemplo, una capilla funeraria (ki-a-nag) donde se enterraba al soberano es denominada, en un texto de época de Lugalanda ${ }^{38}$, ki-a-nag lugal Lagaš ${ }^{\mathrm{ki}}$ (= ki-a-nag de Ur-Nanše ?), mientras que en otro documento ${ }^{39}$ se hace referencia al ki-a-nag ensi $_{2}$ (= ki-a-nag de Enentarzi) ${ }^{40}$.

Las explicaciones a estas alternativas en la utilización de los dos títulos han sido diversas: Para W.W. Hallo ${ }^{4}$, lugal de Lagaš habría sido el título utilizado por Ur-Nanše y Akurgal; inicialmente, también Eanatum lo habría adoptado (Hallo considera que las inscripciones en las que Eanatum se denomina a sí mismo lugal de Lagaš, como por ej. la «Estela de los Buitres» [Ean 1], se datarían al comienzo de su reinado), pero más tarde, debido a sus éxitos militares, prefiere combinar el prestigioso título de lugal de Kiš con el de ensi ${ }_{2}$ de Lagaš; con ello, Eanatum restablecía el modelo político existente en época de Mesilim de Kiš, cuando este rey se denominaba a sí mismo lugal de Kiš y Lugalša'engur era ensi ${ }_{2}$ de Lagaš (Mes 1); los sucesores de Eanatum, según Hallo, habrían retenido el título de ensi ${ }_{2}$ debido al prestigio que Eanatum le había proporcionado, pero no fueron lo suficientemente fuertes como para utilizar el de lugal de Kis;; más tarde, UruKAgina habría decidido asumir nuevamente el título de lugal debido a la pérdida de prestigio del ensi ${ }_{2}$ desde tiempos de Eanatum. La reconstrucción de Hallo, sin embargo, presenta dos dificultades fundamentales: a) Akurgal se denomina a sí mismo ensi (vid. supra); b) no está ni mucho menos clara la datación relativa de la «Estela de los Buitres» $»^{42}$.

37 Esposo de Inanna en Lagaš: sobre este dios, v. G. Selz, «Studies in Early Syncretism: The Development of the Pantheon in Lagaš. Examples for Inner-Sumerian Syncretism», Acta Sumerologica 12 (1990) 117, 121-122.

38 F. Allotte de la Fuÿe Documents ..., n 53:ii.10-11.

39 M.V. Nikolskij, Dokumenty chozjajstvennoj otčetnosti drevnêjšej epochi chaldei iz sobranija N.P. Lichačeva. I, Drevnosti Vostočnyja 3, S. Petersburgo 1908, $\mathrm{n}^{\circ} 161$ = G.J. Selz, Die altsumerischen Wirtschaftsurkunden der Ermitage zu Leningrad, Freiburger Altorientalische Studien 15/1, Stuttgart 1989, n' Nik 161.

40 Cf. M. Lambert, «Le quartier Lagash», Rivista degli Studi Orientali 32 (1957) 130; J. Bauer, «Zum Totenkult im altsumerischen Lagasch», Zeitschrift der Deuschen Morgenländischen Gesellschaft. Suppplementa I (1969) 112; T. Kobayashi, «The ki-a-nag of Enentarzi», Orient 21 (1985) 15-17.

41 W.W. Hallo, Early Mesopotainian ..., pp. 39-44.

42 Cf. J.S. Cooper, Reconstructing History from Ancient Inscriptions: the Lagash-Umma Border Conflict, Sources from the Ancient Near East 2/1, Malibu 1983, pp. 24-26. 
En opinión de M. Lambert ${ }^{43}$, la adopción inicial del título de ensi, ${ }_{2}$ por parte de UruKAgina obedecía - como en el caso de su predecesor - al reconocimiento de la soberanía del rey de Uruk; la muerte de éste, con las consiguientes complicaciones dinásticas, y las pretensiones al trono de Uruk por parte de Lugalzagesi de Umma — ciudad tradicionalmente enemistada con Lagaš por problemas fronterizos, y rey que a la postre se convertiría en el verdugo de UruKAgina-, habrían provocado, según este autor, la decisión de UruKAgina de convertirse en lugal y pretendiente al «imperio» de Uruk. También en este caso, no obstante, la propuesta de Lambert de vincular el título de ensi ${ }_{2}$ a la aceptación de un poder superior en Uruk tiene dos importantes objeciones: a) Eanatum utiliza precisamente el título de ensi ${ }_{2}$ en un momento de expansión militar y de victorias sobre las tropas de ciudades coaligadas ${ }^{44}$; b) Entemena (quien asimismo se denomina ensi $i_{2}$ ), a raíz de su avance militar hacia el $\mathrm{SW}$, consigue poner en peligro la hegemonía de Uruk en esta zona y llega a establecer (Ent 45-73) un tratado de «hermandad» (nam-šeš) con su rey Lugalkinešdudu (= Lugalkiginedudu) $)^{45}$, a quien además se refiere, en la misma inscripción, como ensi de Uruk.

G. Pettinato ${ }^{46}$, por su parte, entiende que en Lagaš (donde la máxima autoridad del Estado se expresaba tradicionalmente con el término ensi ${ }_{2}$ ) los dos títulos pueden ser intercambiables, y no implican, por lo tanto, una jerarquía en la que el ensi ${ }_{2}$ sea de rango inferior, o subordinado, al lugal; sólo en la última fase del Dinástico Antiguo, afirma este autor, asistiremos a un predominio en la utilización del término lugal sobre los otros títulos.

W. Heimpel ${ }^{47}$, finalmente, anota que la vacilación en el uso del título de lugal y ensi, en Lagaš, junto a la utilización casi exclusiva del abstracto nam-lugal, muestran que en esta ciudad no se apreciaba la existencia de una diferencia significativa entre ambos títulos.

La cuestión, ciertamente, no es sencilla. Podría admitirse, en principio, la existencia de dos tradiciones distintas que designan a la máxima autoridad del Estado como ensi ${ }_{2}$ o lugal, y que de ellas terminó por imponerse esta última. Pero esto no nos sirve para explicar por qué, en época presargónica, el soberano de una misma ciudad era unas veces designado como lugal y otras como ensi ${ }_{2}$ (cf. el citado caso de Lagaš, y los de $\mathrm{Adab}^{48}, \mathrm{Kis}^{\mathrm{y}^{49}}$, Umma ${ }^{50}$ y Uruk ${ }^{51}$ ). Tal circunstancia tuvo que obedecer a razones de índole política e ideológica, que aún no estamos en condiciones de comprender en su totalidad, y a determinadas connotaciones de uno y otro título

43 M. Lambert, «La guerre entre Urukagina et Lugalzaggesi», Rivista degli Studi Orientali 41 (1966) 36.

44 Cf. T. Maeda, «'King of Kish' in Pre-Sargonic Sumer», Orient 17 (1981) I1-12; J.S. Cooper, Reconstructing History ..., pp. 24-28. La hegemonía de Eanatum sobre el norte de Babilonia, tras derrotar a una coalición liderada por Akšak (v. por ej. Ean 2:iv.25-v.8, vi.21-vii.2: Ean 3:v.10-vi.5), ha sido, sin embargo, puesta en entredicho por P. Steinkeller, «Early Political Development in Mesopotamia and the Origins of the Sargonic Empire», en M. Liverani (ed.), Akkad: The First World Empire: Structure, Ideology, Traditions, History of the Ancient Near East / Studies 5, Padua 1993, p. 118 n. 20.

45 Cf. J.S. Cooper, Reconstructing History ..., p. 31.

46 G. Pettinato, I Sumeri, pp. 243-245.

47 W. Heimpel, «Herrentum und Königtum ...», pp. 6-8.

48 Eig 1,2; Lum 2; Lugd 1; Med 1; Mes 2.

49 Uhb 1; Mes 1,2 y pass.

50 Url 1; Ent 28 y pass.

51 Ent 45-73; Lukis 1, 2, 5; LuTAR 1; Luzag 1, 3. 
que, sin duda, no fueron siempre las mismas a lo largo de todo el Dinástico Antiguo ${ }^{52}$. Nos encontramos en un período en el que la ciudad-estado se halla inmersa en un proceso que, inexorablemente, conduce a la conformación de realidades políticas de mayor extensión a través de un complicado juego de alianzas y conflictos armados. Sólo un estudio detallado de estas circunstancias (realmente difícil de realizar debido a la escasez de documentación para la mayoría de las ciudades) permitirá comprender el significado real de los términos ensi, y lugal en cada momento y lugar; y explicará, también, por qué UruKAgina decidió convertirse en lugal un año después de su llegada al poder. Es indudable que algo importante en la concepción del poder había cambiado ya en época de este rey: así lo atestiguan la modificación del calendario a partir de su segundo año de reinado, su enfrentamiento a poderosas fuerzas enemigas lideradas por Lugalzagesi y la creación, poco tiempo después, del primer gran imperio mesopotámico con Sargon de Akkad. Incluso desde el punto de vista de la política interna, resulta significativo notar que entre el tercer y cuarto año de su mandato (= Ul 2-3), UruKAgina designó a un ensi ${ }_{2}$ como asistente en determinadas tareas administrativas, especialmente las relacionadas con las propiedades de sus hijos ${ }^{53}$.

En nuestro texto (vii.12-19), por otra parte, la concesión de la nam-lugal a UruKAgina por parte del dios Ningirsu aparece relacionada con su elección entre «36.000 hombres»; este dato -en el que algún autor ha querido ver la confirmación de la ilegitimidad del monarca, e incluso el testimonio de una «elección democrática ${ }^{54}$ - tampoco contribuye gran cosa a aclarar la cuestión de la ilegitimidad de UruKagina, ni el porqué de este nuevo título, pues la misma frase fue utilizada por Entemena (Ent 32:1.2'-3'”), legítimo descendiente de su antecesor ${ }^{55}$.

Sí es razonable suponer, en cualquier caso, que la adopción del título de lugal obtuvo su sanción religiosa en Lagaš, en el segundo año de su reinado (= Ul 1), cuando UruKAgina superó las reticencias iniciales de la clase sacerdotal ${ }^{56}$. En el año siguiente (=Ul 2), además, UruKAgina y su esposa llevaron a Nippur ofrendas de pescado $0^{57} \mathrm{y}$, al menos dos años más tarde $(=\mathrm{Ul} 4)^{58}$, el monarca adquirió el reconocimiento de Nippur: en efecto, el nombre del canal

52 Por ejemplo, el contacto entre el norte y el sur de Babilonia tuvo que ser la causa de significativas modificaciones en las concepciones del poder. Es importante recordar, en este sentido, la influencia que el título de «rey de Kišs» (lugal Kiš) tuvo en el sur de Babilonia, donde fue utilizado, según apunta P. Steinkeller («Early political Development ...», p. 120), como «a generic term that described a particular form of kingship, namely, an autocratic and hegemonistic type that was unknown in the south, one which the southerners associated with the Kishite kingdom».

53 Cl. J. Bauer, «Altsumerische Beiträge (7-9)», Die Welt des Orients 9 (1977-78) 1-3.

54 I.M. Diakonoff, «The Rise of the Despotic State in Ancient Mesopotamia», en I.M. Diakonofl (ed.), Ancient Mesopolamia: Socio-Economic History. A Collection of Sludies by Soviet Scholars, Moscú 1969 (art. aparecido en 1956), p. 184; id., «Some Remarks on the 'Reforms' of Urukagina», Revue d'Assyriologie 52 (1958) 10.

55 J.S. Cooper, Reconstructing History ..., p. 35.

56 E. Sollberger, «L'opposition au pays de Sumer et Akkad», en A. Finet (ed.), La Voix de l'Opposition en Mésopotamie, Bruxelles 1973, p. 33.

57 A. Westenholz, «Diplomatic and Commercial Aspects of Temple Offerings as Illustrated by a Newly Discovered Text», Iraq 39 (1977) 19-21.

58 Los textos de los conos A y B fueron inscritos al menos en el quinto año de reinado de UruKAgina (= U1 4), pues en ellos se menciona la reconstrucción del Antasura (Ukg 1:i.6-9, Ukg 5:i.8-9 = Ukg 4:i.8-9), que se sabe tuvo lugar en ese momento: el nombre de mes de algunos textos (por ej.. F. Allottc de la Fuÿe, Documents ..., n 11 6:xvi.810, $n^{\circ}$ 543:ii. i -2) datados en Ul 4 hace referencia al traslado del dios Ningirsu al nuevo edificio del Antasura. Cf. a este respecto M. Lambert, «La guerre entre Urukagina et Lugalzaggesi», Rivista degli Studi Oriemali 41 (1966) 31-32; F, Carroué, «Le 'Cours-cl'Eau-Allant-ì-NINA ${ }^{\text {ki' } » . ~ A c t a ~ S u m e r o l o g i c a ~} 8$ (1986) 49 n. 42. 
registrado en xii.12, «Ningirsu-es-Soberano-ante-Nippur», muestra la aceptación, por parte de Nippur y su gran dios Enlil, de UruKAgina y Ningirsu, el dios de su ciudad".

\section{OTRAS CUESTIONES SOBRE LAS CIRCUNSTANCIAS DE LA LLEGADA AL PODER DE URUKAGINA}

De la misma forma que, inicialmente, UruKAgina adopta una política continuista en su titulatura con relación a sus antecesores, para después romper con ella y asumir la condición de lugal una vez que adquiere la aceptación del mundo divino (o sacerdotal), también se observa un viraje similar en el hecho siguiente: en una inscripción sobre un gozne de puerta (Ukg 12) que dataría de comienzos de su reinado, UruKAgina nos dice que su dios protector es Sulutul, el dios personal de la dinastía de sus antecesores; más tarde, sin embargo, su dios personal se (re)convertirá en Ninšubur (Ukg 7:ii'.6-7; Ukg 10:iv.10-v.1) ${ }^{60}$.

La relación con Lugalanda es, asimismo, una cuestión muy interesante, que abunda, además, en la voluntad de UruKAgina por llevar cabo una transición lo menos dolorosa posible. Así, sabemos que Lugalanda sobrevivió a la llegada al poder de UruKAgina, y que murió durante el segundo año de gobierno de éste $(=\mathrm{Ul} \mathrm{l})^{61}$. Y cuando, durante su tercer año de reinado (= Ul 2), Baragnamtara (esposa de Lugalanda) muere, Šǎ̌a (esposa de UruKAgina) organiza para ella unos espléndidos funerales en los que toman parte al menos 617 personas, entre ellas 164 cantantes (gala) y 118 plañideras (dam-ab-ba) ${ }^{62}$. Por ello, es de suponer que el ascenso al poder de UruKAgina no fue traumático, aunque, de hecho, desconocemos sus mecanismos: la clave quizás esté en la figura de Baragnamtara, de quien se ha dicho que la deferencia tenida hacia ella se debió a su relevancia como antigua administradora del $\mathrm{E}_{2} \cdot \mathrm{MI}_{2}^{63}$. Es posible también, no obstante, que hubiese existido algún tipo de parentesco entre Saša y Baragnamtara, lo que podría explicar las dimensiones de sus funerales y una transición fácil entre Lugalanda y UruKAgina; por el momento, sin embargo, tal hipótesis, no pasa de ser una mera especulación ${ }^{G 4}$.

En la misma línea de contemporizar inicialmente con la figura y tradiciones de sus predecesores, se situaría la actitud de UruKAgina hacia el culto a los reyes muertos. Según ha demos-

59 M. Lambert, «La guerre entre Urukagina...», pp. 39-40; F. Carroue, «Le 'Cours-d'Eau-...», p. 49 n. 43. Es asimismo significativo (cf. M. Lambert, «La guerre entre Urukagina...», p. 40) el antropónimo UruKAgina-Enlile-su «Enlil reconoce a UruKAgina» en M.V. Nikolskij, Dokumenty ..., n ${ }^{\circ} 16: \mathrm{ix} .10=\mathrm{G} . \mathrm{J}$. Selz, Die alisumerischen ..., $\mathrm{n}^{\circ}$ Nik 16:ix.10; F. Allotte de la Fuÿe, Documents ..., n 116:ix.10, n 117:ix.2, $\mathrm{n}^{\circ} 118: \mathrm{ix} .6$; de estos textos los tres primeros están datados en el cuarto año de UruKAgina (Ul 4).

60 E. Sollberger, «L'opposition ...», p. 33.

61 Cf. A. Deimel, «Die Relormtexte ...», pp. 32-51; J. Bauer, «Altsumerische Beiträge ...», p. 3; T. Kobayashi, «The ki-a-nag ...», p. 21.

62 H. de Genouillac, Tablettes sumériennes archaiques, París 1909, n 9; W. Förtsch, Allbabylonische Wirtschaftstexte aus der Zeit Lugalanda's und Urukagina's, Vorderasiatische Schriftdenkmäler der Königlichen Museen zu Berlin 14/I, Leipzig 1916, n $137=\mathrm{J}$. Bamer, Altsumerische Wirtschaftstexte aus Lagasch, Roma $1972,17^{\circ} 66$.

63 Por ej., J.M. Asher-Greve, Frauen ..., p. 151.

64 Nótese, además, que, como apunta J. Bauer ( Zum Totenkult ...», pp. 107, 113 n. 17), es probable que las hijas de Baragnamtara y Lugalanda ( $\mathrm{MI}_{2}$-šaga y Geme-Nanše), hubiesen recibido un «subsidio», por medio de Šša, tras la llegada de UruKAgina al poder; $\mathrm{MI}_{2}$-šaga, sin embargo, murió muy joven, durante el segundo año de UruKAgina (= UI l): v. J.M. Asher-Greve, Fraten ..., p. 153. 
trado T. Kobayashi ${ }^{65}$, los reyes de Lagaš fundadores de una dinastía construyeron una capilla funeraria (ki-a-nag) destinada a su propio enterramiento, al de su esposa y al de los sucesivos gobernantes de dicha dinastía; esto probablemente ocurrió con Ur-Nanše, y, con seguridad, con Enentarzi: en el ki-a-nag de Enentarzi, construido durante su primer año de reinado, fueron enterrados él mismo, su esposa Dimtur, y Lugalanda y Baragnamtara. UruKAgina, probablemente obligado todavía por las circunstancias de su llegada al poder, necesitado de llevar a cabo esa transición no traumática a la que aludíamos, continuó llevando ofrendas al ki-a-nag de Enentarzi durante su primer año como ensi ${ }_{2}$. Sin embargo, una vez proclamado lugal, dejó de hacerlo y se construyó su propio ki-a-nag, emblema de la realeza, en el cual, además, introdujo el culto a sus antepasados con el fin de fortalecer su figura de rey incontestable.

\section{EL CONTEXTO HISTÓRICO}

En definitiva, la no pertenencia de UruKAgina a la dinastía precedente pudo propiciar, en cierta medida, la promulgación de unas «reformas» que pretendían contentar a diversos sectores sociales, especialmente el sacerdotal, mediante el simple recurso a la descalificación de las administraciones anteriores. Estas «reformas», sin embargo, no modificaron de manera sustancial el modelo económico y religioso en vigor, y fueron, además, formuladas en un momento en el que UruKAgina se sentía ya lo suficientemente fuerte como rey, y política y moralmente distanciado de los reyes precedentes.

Por ello, las razones para la redacción de nuestro texto -cuya comprensión es, al fín y al cabo, lo que más interesa para poder valorarlo en su justa medida- hay que buscarlas también en otros ámbitos más o menos inmediatos a las circunstancias políticas del momento. En este sentido, un factor que pudo tener, asimismo, alguna influencia fue el enfrentamiento con Lugalzagesi de Umma.

El conflicto entre las ciudades de Lagaš y Umma tiene una larga historia que arranca, según nos cuentan los mismos textos de Lagaš, desde la época de Mesilim de Kiš (Ean 6, 63; Ent 2829), y que se prolongó incluso hasta los tiempos de la Tercera Dinastía de Ur ${ }^{66}$. Los detalles de este enfrentamiento nos son especialmente bien conocidos, desde el punto de vista de Lagaš, gracias a las referencias que de él dieron sobre todo Eanatum, Entemena y UruKAgina. De sus relatos se desprende que en la lucha se vieron también implicadas, de forma progresiva, otras ciudades del sur mesopotámico en un proceso que culminaría con la victoria de Lugalzagesi sobre UruKAgina. Lugalzagesi, originario de Umma, partiendo del control que ya ejercía Uruk sobre parte de Sumer y de la estrecha relación -quizás familiar- existente entre las casas reales de Umma y Uruk, había conseguido aglutinar en torno a sí a algunas ciudades sumerias en su lucha contra Lagaš ${ }^{67}$. Desde esta posición comenzó un acoso a UruKAgina, probablemen-

65 T. Kobayashi, «The ki-a-nag ...».

66 Sobre la historia del conflicto, v. G. Pettinato, «I 'Frontiera Divina' e la sua soluzione durante la Terza Dinastia di Ur», Mesopotamia 5-6 (1970-71) 281-320; J.S. Cooper, Reconstructing History ...; G. Steiner, "Der Grenzvertrag zwischen Lagas und Unma", Acta Sumerologica 8 (1986) 219-300.

67 Las dimensiones precisas de esta alianza nos son desconocidas, aunque J.S. Cooper (Reconstructing History ..., p. 35) afirma que sLagash at this time was under pressure from a Sumer united under the leadership of Uruk, which leadership had strong, possibly familial ties to the local ruler at Umma». 
te ya desde los comienzos del reinado de éste, que alcanzó su máxima intensidad a partir del quinto año de UruKAgina (= Ul 4$)^{68}$. Dado que esta es la fecha post quem el texto de Las «Reformas»... fue redactado (v. nota 58), no resulta difícil pensar que su promulgación obedeciera también al deseo del rey de atraerse a la población en un momento de graves dificultades en el exterior ${ }^{69}$.

Pero al margen de la utilización de Las «Reformas»... como arma política, sea por cuestiones internas o por problemas en el exterior, el texto, según ha mostrado M. Liverani ${ }^{70}$, revela también la existencia de un estrato de la población obligado a endeudarse y a ceder sus propiedades e hijos al acreedor con el fin de poder satisfacer los intereses de su deuda; en el caso de que ésta no pudiera ser satisfecha - algo que ocurría con frecuencia-, el mismo deudor se veía en la situación de tener que ofrecer sus servicios a su acreedor, renunciando así a su condición de «trabajador libre». Las circunstancias que desencadenaban este proceso -que afectaba a una parte importante de la población y se repetía periódicamente- se encontraban tanto en una inseguridad climática que ponía constantemente en peligro el rendimiento de las cosechas, como en la frecuente desprotección del campesinado libre ante los vaivenes políticos y militares y en la marginación de sus tierras respecto a las de las grandes organizaciones públicas. La única manera que se conocía para corregir estas situaciones de crisis era «restituyendo la libertad a los ciudadanos de Lagaš que estaban endeudados» (xi.20-21,28-29), y, si era posible, como en el caso de UruKAgina, culpando de ello a las administraciones anteriores.

Finalmente, Las «Reformas»... encuentran todo su sentido en un contexto de desintegración progresiva de la ciudad-estado como modelo político dominante en el sur de Mesopotamia. Como bien ha hecho ver H.J. Nissen ${ }^{71}$, el pronunciado particularismo de las ciudades de Babilonia se debía, en gran medida, a la necesidad de delimitar claramente un área de influencia desde la que poder controlar los sistemas de regadío de los que dependía la vida económica; tal circunstancia contribuyó, además, a la consolidación del concepto de un dios tutelar de la ciudad. Contra la fragmentación surgió, sin embargo, la tendencia a la cooperación y la concentración de fuerzas: así lo exigían las inevitables necesidades comerciales y la interdependencia creciente de todos los sistemas locales de irrigación vinculados al Éufrates ${ }^{72}$. En dicho contexto hay que explicar la aparición de monarcas que pretendían erigirse en árbitros de conflictos interciudadanos o en hegemónicos en Sumer (Mesilim de Kiš, Eanatum de Lagaš, Lugalzagesi de Umma). Y es también en esta dinámica de particularismo $v s$. centralismo donde hay que situar la figura de UruKAgina y el significado de sus «reformas»: con éstas, apunta Nissen, el rey pretendía aglutinar las fuerzas de la ciudad en torno a la figura de su dios en un último e innovador intento por preservar el modelo político de ciudad-estado. Para ello, UruKAgina formuló el principio de que la ciudad, junto con sus habitantes y tierras, eran de hecho propiedad del dios; esta teoría — que en nuestro texto se intenta legitimar apelando a una ficticia

68 Cf. M. Lambert, «La guerre entre Urukagina ...», pp. 40-42; J.S. Cooper Reconstructing History ..., pp. 3436.

69 Cf. M. Lambert, «La guerre entre Urukagina ...», p. 36.

70 M. Liverani, Antico Oriente ..., pp. 199-200.

71 H.J. Nissen, The Early History ..., pp. 140-149.

72 Otro elemento que habría que tomar también en consideración es la influencia que las instituciones políticas del norte de Babilonia (en particular el modelo de realeza de la ciudad de Kišs) ejercieron sobre las ciudades-estado del sur: sobre este aspecto véase P. Steinkeller, «Early Political Development ...», pp. 120-129. 
tradición (vii.20-22) - no puede, sin embargo, extrapolarse sin más a períodos anteriores de la Historia de Mesopotamia y considerar que la «ciudad-templo» ideada por UruKAgina (que además no llegó a tener una realización práctica) era el tipo de entidad política generalizada desde la época de Uruk.

Se entiende también así que los sacerdotes y templos, estandartes de la ideología defendida por UruKAgina, aparezcan particularmente protegidos en el texto de Las "Reformas».., y que Lugalzagesi se ensañe en su destrucción en uno de los episodios finales de su lucha contra el rey de Lagaš (Ukg 16). Al final de la misma inscripción que describe estos hechos, UruKAgina pronunciará unas palabras que revelan claramente cómo su concepto de que el rey era sólo el instrumento de la voluntad del dios no era precisamente compartido por Lugalzagesi: «iQue Nisaba, la diosa de Lugalzagesi, le haga llevar su pecado sobre su cuello!» (Ukg 16:viii.11ix. 3$)^{73}$

\section{EL IDEAL DE JUSTICIA}

La última cuestión que quedaría por considerar es la relación del texto de Las «Reformas»... con las inscripciones reales conocidas como «códigos». Un precedente cercano a la acción de UruKAgina de conceder la libertad (ama-al-gi ${ }_{4}$ ) a los ciudadanos de Lagaš lo encontramos en dos inscripciones conmemorativas de Entemena (Ent 35 y 79); en ellas, con una fraseología similar a la empleada por UruKAgina en el texto de Las «Reformas»..., Entemena recuerda cómo proclamó la libertad en Lagaš (y otras ciudades [Ent 79:v.4-8]), «devolviendo el hijo a la madre y la madre al hijo» (Ent 35:v.2-6; Ent 79:iii.10-iv.3), y anulando las obligaciones relacionadas con préstamos a interés de grano (Ent 79:iv.4-5). Como Z. Yang ha puesto de manifiesto ${ }^{74}$, los textos de Entemena tratan todavía el tema de la «liberación» y la «justicia» de una forma secundaria, permaneciendo en primer plano, como en la mayoría de las inscripciones reales de la época, las actividades edilicias del monarca. El texto de UruKAgina, en cambio, aunque utiliza como pretexto la construcción de templos y canales, se centrará por primera vez en la descripción y objetivos de unas «reformas» cuyo fin es también demostrar su calidad de «ey justo».

Tras el texto de Las «Reformas»..., pasarán más de doscientos años hasta que volvamos a encontrar, ya plenamente desarrollado, un nuevo género literario que habitualmente se denomina "código»: se trata del "Código de Ur-Namma» ${ }^{75}$. Según el estudio de Yang, los elementos que permanecen constantes en este texto en relación al de UruKAgina son: 1) la inclusión de

73 Cf. H. Vanstiphout, «Political Ideology in Early Sumer», Orientalia Lovaniensia Periodica 1 (1970) 36-37; H.J. Nissen, The Early History ..., pp. 148-149.

74 Z. Yang, "King of Justice», Aula Orientalis 9 (1991) =Fs. M. Civil, pp. 243-249.

75 Sobre la posibilidad de que hubiese sido Sulgi el verdadero autor del «Código de Ur-Namma», v.: J.van Dijk, en F.Yildiz, "A Tablet of Codex Ur-nammu from Sippar», Orientalia NS 50 (1981) 93-94 n. 20a; S.N. Kramer, «The Ur-Nammu Code: Who was its Author?», Orientalia NS 52 (1983) 453-456; P. Steinkeller, "The Administrative and Economic Organization of the Ur III State: The Core and the Periphery», en McG. Gibson - R.D. Biggs (edd.), The Organization of Power. Aspects of Bureatcracy in the Ancient Near East, Studies in Ancient Oriental Civilization 46, Chicago 1987, pp. 19-20 n. 1 y p. 21 n. 10; P. Michalowski - C.B.F. Walker, «A new Sumerian 'Law Code'», en H. Behrens - D. Loding - M.T. Roth (edd.), DUMU-E-DUB-BA-A, Stıdies in Honor of Ake Sjöberg, Occasional Publications of the Samuel Noah Kramer Fund 11, Philadelphia 1989, pp. 384-386. 
acontecimientos históricos; 2) la utilización de ejemplos para demostrar que el rey gobernaba con justicia. Las novedades, por el contrario, radicarían en: 1) la utilización de toda una sección de oraciones condicionales, probablemente recogidas de la práctíca legal consuetudinaria, para demostrar que el reinado de Sulgi (autor del "Código de Ur-Namma») era un reinado de justicia; 2) La utilidad del texto en sí, el cual había dejado de ser una inscripción conmemorativa para convertirse en un monumento independiente redactado mediante un nuevo tipo de composición literaria. Así pues, mientras UruKAgina demostraba su justicia mediante la promulgación de determinadas medidas, Śulgi lo hizo velando por el mantenimiento de las prácticas legales basadas en el derecho consuetudinario. En los posteriores - y más elaborados- "códigos» de Lipit-Ištar y Hammurapi, el papel ejercido por el rey sobre el sistema legal será cada vez más activo, pero no se llegaría en ningún caso a desarrollar un auténtico «código de leyes». Lo que sí permanecerá en todos ellos, desde la época de UruKAgina, es el deseo de los monarcas de mostrar a los ciudadanos su condición de «reyes justos».

II

\section{LAS «REFORMAS» DE URUKAGINA ${ }^{76}$}

\begin{tabular}{|c|c|c|}
\hline i & 1 & ${ }^{\mathrm{d}} \mathrm{Nin}-\mathrm{gir}_{2}-\mathrm{su}$ \\
\hline & 2 & ur-sag ${ }^{d}$ En- $1 i_{2}-1 a_{2}$-ra \\
\hline & 3 & Uru-KA-gi-na \\
\hline & 4 & lugal \\
\hline & 5 & Lagaš $^{\mathrm{ki}}-\mathrm{ke}_{4}$ \\
\hline & 6 & $e_{2}$-gal Ti-ra-ašs \\
\hline & 7 & mu-na-du \\
\hline & 8 & An-ta-sur-ra \\
\hline & 9 & mu-na-du ${ }_{3}$ \\
\hline $\mathbf{i i}$ & 1 & $\mathrm{e}_{2}{ }^{\mathrm{d}} \mathrm{Ba}-\mathrm{ba}_{6}$ \\
\hline & 2 & mu-na-du \\
\hline & 3 & Bur-sag \\
\hline & 4 & $e_{2} s a_{2}-$ dug $_{4}-k a-n i$ \\
\hline & 5 & mu-na-du \\
\hline & 6 & $\mathrm{ga}_{2}$ udu ur${ }_{4}$ \\
\hline
\end{tabular}
A Ningirsu, héroe de Enlil, UruKAgina", rey de Lagaš, el palacio de Tira'aš ${ }^{78}$ le construyó; el Antasura ${ }^{79}$ le construyó; el templo de Baba le construyó; el Bursag, su santuario para las ofrendas regulares, le construyó; su edificio de Urukug en el que se esquilan las ovejas

76 El texto transliterado y traducido corresponde a Ukg 5 (cono C); las variantes de Ukg 4 (cono B) se anotan a pie de página.

77 Sobre la lectura del nombre de este rey, v. W.G. Lambert, "The Reading of the Name uru.KA.gi.na», Orientalia NS 39 (1970) 419; D.O. Edzard, «rrikagina (Urukagina)», Aula Orientalis $9(1991)=$ Fs. M. Civil, pp. 77-79; P. Steinkeller, «The Reforms of UruKAgina ...», p. 227 n. 2; W.G. Lambert, «The Reading of Uru-KA-gi-na Again», Aula Orientalis 10 (1992) 256-258.

78 Tira' aš = santuario de Ningirsu en las cercanías de Girsu: D.O. Edzard - G. Farber - E. Sollberger, Die Orts und Gewässernamen der präsargonischen und sargonischen Zeit, Répertoire Géographique des Textes Cunéiformes 1 , Wiesbaden 1977, pp. 159-160.

79 Antasura = localidad de la zona fronteriza entre Umma y Lagaš: D.O. Edzard - G. Farber - E. Sollberger, Die Orts- und Gewässernamen ..., pp. 15-16. 


\begin{tabular}{|c|c|c|}
\hline 7 & Uru-ku ${ }_{3}$-ga-ka-ni & \\
\hline 8 & mu-na-du & le construyó; \\
\hline 9 & dNanše & para Nanše \\
\hline 10 & $\mathrm{id}_{2}-\mathrm{NINA}^{\mathrm{ki}}$-gin & el «Canal-que-fluye-hacia- NINA» ${ }^{80}$, \\
\hline 11 & $\mathrm{id}_{2} \mathrm{ki}^{-\mathrm{ag}_{2}}-\mathrm{ga}_{2}-\mathrm{ni}^{81}$ & su amado canal, \\
\hline 12 & al mu-na-du & le excavó \\
\hline 13 & kun-bi & (y) su desembocadura \\
\hline 14 & $a b \stackrel{s}{s} a_{3}-g a$ & hasta el interior de (la región de) Hुōr \\
\hline 15 & mu-na-ni-la & se lo hizo llegar \\
\hline 1 & $\operatorname{bad}_{3} \mathrm{Gir}_{2}-\mathrm{su}_{2}^{\mathrm{ki}} 83$ & los muros de Girsu \\
\hline 2 & mu-na-du & le construyó. \\
\hline 3 & ud ul-li 2 -a-ta & Desde los lejanos días, \\
\hline 4 & numun $\mathrm{e}_{3}-\mathrm{a}-\mathrm{ta}$ & desde el surgimiento de la semilla, \\
\hline 5 & ud-bi-a & en aquellos días, \\
\hline 6 & $l u_{2}-m a_{2}-l a h_{5}-k_{4}$ & el «hombre de la barca» \\
\hline 7 & $\mathrm{ma}_{2} \mathrm{e}-\mathrm{dab}_{5}$ & se apropiaba de las barcas; \\
\hline 8 & anše $u_{2}$-du-le & de los asnos el administrador de los rebaños \\
\hline 9 & e-dab 5 & se apropiaba; \\
\hline 10 & udu $u_{2}$-du-le & de las ovejas el administrador de los rebaños \\
\hline 11 & e-dab ${ }_{5}$ & se apropiaba; \\
\hline 12 & $u_{3}-\operatorname{sar} u_{2}-m_{11}$ & de ..... \\
\hline 13 & enku-re ${ }_{6}$ & el supervisor de la pesca \\
\hline 14 & $e-d_{a b}$ & se apropiaba; \\
\hline 15 & gudu $_{4}$-ge-ne & los sacerdotes-gudug ${ }^{84}$ \\
\hline 16 & se gub-ba & las contribuciones de cebada \\
\hline 17 & Ambarki-a & en Ambar $^{85}$ \\
\hline 18 & $\mathrm{e}-\mathrm{ag}_{2}$ & medían $^{86}$ \\
\hline
\end{tabular}

80 Sobre este canal y el territorio de NINA (= Surghul), v. F. Carroué, «Le 'Cours-d'Eau-...», pp. 13-57.

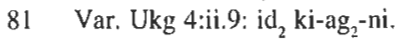

82 Sobre la región de Hōr (a-ab, a-ab-ba, ab), v. H. Waetzoldt, «Zu den Strandverschiebungen am Persischen Golf und den Bezeichnungen des Hōrsm, en Rupert Carola, Sonderheft Strandverschiebungen (Heidelberg 1981), pp. 168-169. Sobre los trabajos de canalización v., para este período, T. Maeda, «Work Concerning Irrigation Canals in PreSargonic Lagash», Acta Sumerologica 6 (1984) 33-53; de forma más específica están tratados los datos concernientes a este texto en B. Hruška, «Die Bewässerungsanlagen in den altsumerischen Königsinschriften von Lagaš», Bulletin on Sumerian Agriculture 4 (1988) 65.

83 Var. Ukg 4:ii.14: bad $_{3} \mathrm{Gir}_{2}-\mathrm{su}^{\mathrm{k}_{1}}$.

84 Los gudug eran sacerdotes de baja categoría que estaban sujetos al pago de tasas de cebada según se desprende de otros textos (por ej. W. Förtsch, Altbabylonische Wirtschaftstexte ..., n ${ }^{\circ} 170=\mathrm{J}$. Bauer, Altsumerische Wirtschafistexte ..., $\mathrm{n}^{\circ}$ 7:iii.5): J.S. Cooper, Presargonic Inscriptions. Sumerian and Akkadian Royal Inscriptions I, New Haven 1986, p. 73. En Ukg 6:i.7'-9' se añade que los sacerdotes-gudug tenían que construir un almacén para esas tasas de cebada.

85 Ambar = zona de la ciudad-estado de Lagaš: D.O. Edzard - G. Farber, Die Orts- und Gewässernamen der 3. Dynastie von Ur, Répertoire Géographique des Textes Cunéiformes 2, Wiesbaden 1974, pp. 7-8; D.O. Edzard - G. Farber - E. Sollberger, Die Orts- und Gewässernamen ..., pp. 11-12.

86 Este pasaje es discutido e interpretado de la siguiente manera por B. Foster, «A New Look ...», p. 234: «the barley rentals (paid on leased fields of the Great Household) were measured out in the (region called) Marshland by the Shutug-priests». 


\begin{tabular}{|c|c|c|}
\hline & 19 & sipa udu-sig $-k a-k e_{4}-n e$ \\
\hline & 20 & bar adu had 2 -ka \\
\hline iv & 1 & $\mathrm{ku}_{3}$ bi-gar-re ${ }_{2}-\mathrm{es}_{2}$ \\
\hline & 2 & $\mathrm{lu}_{2}-\mathrm{es} \mathrm{s}_{2}-\mathrm{gid}_{2}$ \\
\hline & 3 & gala-mah \\
\hline & 4 & agrig \\
\hline & 5 & $\mathrm{lu}_{2}$-bappir ${ }_{3}$ \\
\hline & 6 & ugula-ugula-ne \\
\hline & 7 & bar sila - gaba-ka-ka \\
\hline & 8 & $\mathrm{ku}_{3}$ bi-gar-1-e $-\mathrm{es}_{2}$ \\
\hline & 9 & gud dingir-re - -ne-ke ${ }_{4}$ \\
\hline & 10 & ki sum-ma \\
\hline & 11 & ensi ${ }_{2}-k a$ \\
\hline & 12 & $\mathrm{i}_{3}-\mathrm{uru}_{4}$ \\
\hline & 13 & $\mathrm{GANA}_{2} \mathrm{sa}_{6}-\mathrm{ga}$ \\
\hline & 14 & dingir-re ${ }_{2}-n e-k a$ \\
\hline & 15 & ki sum-ma \\
\hline & 16 & ki ukuš ${ }_{2}$ \\
\hline & 17 & ensi $i_{2}-\mathrm{ka}$ \\
\hline & 18 & e-gal -lam \\
\hline & 19 & anse bir $_{3}-\mathrm{ra}$ \\
\hline & 20 & gud $d u_{7}-d u_{7}$ \\
\hline & 21 & sanga-sanga-ne \\
\hline & 22 & e-ne-keš $-\mathrm{ra}_{2}-\mathrm{am}_{6}$ \\
\hline & 23 & še sanga-sanga-ne \\
\hline & 24 & eren $_{2}$ ensi $_{2}-\mathrm{ka}-\mathrm{ke}_{4}$ \\
\hline & 25 & e-ba \\
\hline $\mathbf{v}$ & 1 & lug2PI dNin-KILIM ${ }^{\text {gi4-li2-na }}$ \\
\hline & 2 & $\operatorname{tug}^{2} \mathrm{U}_{3} \cdot \mathrm{AS}_{2}$ \\
\hline & 3 & $\operatorname{tug} 2{ }^{2} \mathrm{Su}^{3} \mathrm{du}_{8}-\mathrm{ur}_{3}$ \\
\hline & 4 & nig -bar-ba $^{88}$ \\
\hline & 5 & gada- $U_{3} \cdot \mathrm{LA}_{2}$ \\
\hline & 6 & gu-su 3 -ga \\
\hline & 7 & gu-sa-la \\
\hline & 8 & sag-šu $u_{4}$ zabar \\
\hline
\end{tabular}

los pastores de ovejas de lana

por una oveja pura

pagaban plata;

el agrimensor

el cantante superior del culto,

el administrador,

el maestro cervecero,

todos los supervisores

por un cordero lechal

pagaban plata ${ }^{87}$;

los bueyes de los dioses

en los campos de cebollas

del ensi ${ }_{2}$

araban;

sobre los buenos campos

de los dioses

las plantaciones de cebollas

(y) las plantaciones de pepinos

del ensi ${ }_{2}$

estaban;

las cuadrillas de asnos

(y) los vigorosos bueyes

de los administradores del templo

eran uncidos,

(pero) la cebada de los administradores del templo

por el personal del ensi ${ }_{2}$

era recibida;

un vestido-... de mangosta,

un vestido-...,

un vestido lujoso (?),

un vestido-...,

un vestido-... de lino,

un ... de fibra de lino,

fibra de lino atada en haces,

un yelmo de bronce,

87 iii.19-iv.8: pasaje que probablemente hace referencia a una tasa que había que pagar por el ganado nacido en campos de alquiler donde pastaban los animales del arrendatario: P. Steinkeller, «The Renting of Fields ...», pp. 132133. B. Foster, "A New Look ...», p. 232, por su parte, discute estas líneas rebatiendo las interpretaciones de otros autores (A. Deimel, A. Poebel, M. Lambert y S.N. Kramer), y propone la siguiente traducción: «The sheperds of woolbearing sheep would put down the (price in) silver on account of a white sheep; the surveyor, chief cantor, steward, brewer, and foreman would put down the (price in) silver on account of a GABA-sheep». Este pasaje (junto a viii.6-10) también ha sido recientemente discutido por D. A. Foxvog, "A New Lagaš Text ...", pp. 11-15, quien propone la interpretación de udu had ${ }_{2}$ (iii.20) como "oveja pura", y matiza el significado del verbo gar (iv.8) como "cargar (en la cuenta de alguien)"; en este artículo, además, el autor publica un texto administrativo de época de Lugalanda relacionado con el "abuso" al que alude UruKAgina en este pasaje.

88 Var. Ukg 4:v.7: ${ }^{\text {ug }}{ }^{2}$ nig $_{2}$-bar-ba. 
un clavo de bronce,

10 ru-ur-ra-zabar

11 kuš zalag-ga

$12 \mathrm{a}_{2}$-buru ${ }_{4}-\mathrm{gi}^{\text {inusen }}$

13 KU.MUL

14 BLL $_{2}$.GI.ŠE.DUL

15 maš-bar-dul ${ }_{s}$

16 sanga-sanga-ne

$17 \quad \mathrm{IL}_{2}-\mathrm{Se}_{3}$

$18 \quad \mathrm{i}_{3}-\mathrm{IL}_{2}-\mathrm{am}_{6}$

19 sanga-GAR-ke

20 kiri ${ }_{6}$ ama-uku $_{2}-\mathrm{ra}_{2}$

21 giš na ba-ni-ri-ri

22 gi-lam

23 e-ta-kešs $-\mathrm{ra}_{2}$

24 adda ki-mah-še ${ }_{3}$ DU ${ }^{92}$

25 kas-ni 7 dug

26 ninda-ni 420-nam

272 (ul) še-ha-zi ${ }^{93}$

281 tug $_{2}$

291 maš-sag-ga

vi 11 gišs $\mathrm{na}_{2}$

$2 \mathrm{uh}_{3}$-muš ${ }_{3}$-e

3 ba-DU

41 (ul) še lu $u_{2}$-umum-ma-ke ${ }_{4}$

5 ba-DU

6 gi-`En-ki-ka-ka

$7 \quad \mathrm{lu}_{2} \mathrm{u}_{3}-\mathrm{DU}$ un ... de bronce,

una piel brillante,

plumas de «cuervo que grazna»,

(y) una cabra.$^{89}$

los administradores del templo

como contribución-IL $\mathrm{IL}_{2}$

entregaban;

los administradores-GAR del templo $0^{90}$

en el huerto de un ama-uku ${ }_{2}{ }^{91}$

talaban árboles

(y) los frutos

recogían;

para llevar un cadáver al cementerio

siete jarras de cerveza,

420 panes,

2 ul (72 l.) de cebada-ha-zi,

un vestido,

un carnero-guía,

(y) una cama

el $\mathrm{uh}_{3}$ - mus $_{3}{ }^{94}$

cogía para sí,

(y) el lu $\mathrm{l}_{2}$-umum-ma 1 ul (36 l.) de cebada

cogía para sí;

cuando a la «caña de Enki»»6

un hombre era llevado

89 v.1-15: sobre estos objetos, v. comentario en Steible, Die altsumerischen ... II, pp. 145-147.

90 Sobre este tipo de sacerdotes, v. B. Hruška, «Die innere Struktur ...», p. 111; F. Pomponio, La prosopografia dei testi presargonici di Fara, Studi Semitici NS 3, Roma 1987, p. 309.

91 ama-uku $_{2}$ ( $v .20, x .18$ ): término (con frecuencia traducido como "pobre») que designa a una categoría de personas. Faltan, sin embargo, paralelos para definir sus características, las cuales no pueden conocerse sólo a través de la etimología del término (ama «madre», uku ${ }_{2}$.r ac. lapnu «pobre»): v. discusión en B. Hruška, «Die innere Struktur ...», p. 111.

92 Var. Ukg 4:vi.4: ki-mah KI DU.

93 Var. Ukg 60:ii'.2': [še]-ha-zi-na.

94 uh $_{3}$-muš s $_{3}$ vi.2,14, viii.37, ix.7): categoría de personas de estatus relativamente alto; en F. Allotte de la Fuÿe, Documents ..., $\mathrm{n}^{\circ} 226: v i .7^{\prime}$ y $\mathrm{n}^{\circ} 216$ :iii.5 aparecen al mismo nivel que la esposa del sanga (B. Hruška, "Die innere Struktur ...», p. 113).

$95 \mathrm{lu}_{2}$-umum-ma (vi,4,17, viii.39, ix.9): categoría de personas de características no definidas. B. Hruška, «Die innere Struktur ...», p. 113 propone, en base a este contexto, un posible significado de «embalsamador» 0 «sepulturero».

96 En opinión de Bauer, «Altsumerische Beiträge ...», p. 9, la «caña de Enki» designaría un lugar donde se celebraban rituales de curación; Steible, Die altsumerischen ... Il, p. 148 apunta también la posible relación de este lugar con el «santuario de Girsu»: el tex to Urn 49 comienza con una alabanza a la caña y concluye con una descripción de la construcción del santuario, la cual ha de ser decidida por Enki; T. Kobayashi, «The ki-a-nag ...», p. 26 n. 3, lo identifica con un lugar de enterramiento: «hole (tomb) of Enki». 
8 kas-ni 7 dug

9 ninda 420-nam

102 (ul) še

111 tug $_{2}$

121 gis $\mathrm{na}_{2}$

131 giš dur ${ }_{2}$-gar

$14 \mathrm{uh}_{3}$-muss $-\mathrm{e}$

15 ba-DU

161 (ul) še

$17 \quad \mathrm{lu}_{2}$-umum-ma-ke

18 ba-DU

19 giš-kin-ti

20 ninda-\$u-il $-\mathrm{la}$

$21 \mathrm{i}_{3}$-tuku-am,

22 guruš-MIN-me

23 addir a-bul - -la

24 i $_{3}$-tuku-am 6

$25 \mathrm{e}_{2}$ ensi $\mathrm{i}_{2} \mathrm{ka}$

26 GANA $_{2}$ ensi $-\mathrm{ka}_{2}-\mathrm{ke}_{4}$

$27 \mathrm{e}_{2} \mathrm{E}_{2} \cdot \mathrm{MI}_{2}{ }^{99}$

$28 \mathrm{GANA}_{2} \mathrm{E}_{2} \cdot \mathrm{MI}_{2}-\mathrm{ke}_{4}$

29 e, nam-dumu

30 GANA, nam-dumu-ke ${ }_{4}$

$31 \mathrm{za}_{3} \mathrm{i}_{3}-\mathrm{us}_{2}-\mathrm{us}_{2}-\mathrm{am}_{6}$

32 ki-sur-ra

33 dNin-gir ${ }_{2}$ su-ka-ta

34 a-ab- $\mathrm{se}_{3}$

35 maškim-di

36 e-gal -lam

37 ŠUB-lugal-ke,
7 jarras de cerveza,

420 panes,

2 ul (72 1.) de cebada,

un vestido,

una cama,

(y) un asiento de madera

el $\mathrm{uh}_{3}$-mus $\mathrm{s}_{3}$

cogía para sí,

(y) 1 ul (36 1.) de cebada

el lu ${ }_{2}$-umum-ma

cogía para sí;

los artesanos

el ninda-šu-il ${ }_{2}-\mathrm{la}^{97}$

tenían,

y las parejas de obreros ${ }^{98}$

la tarifa de «la puerta de la ciudad»

tenían;

en las propiedades del ensi ${ }_{2}$

y en los campos del ensi,

en las propiedades de la "Casa de la Mujer"»

y en los campos de la «Casa de la Mujer»,

en las propiedades de los hijos

y en los campos de los híjos

se cometían irregularidades ${ }^{100}$;

desde la frontera

de Ningirsu

hasta (la región de) Hūor

había gente que en calidad de inspectores

actuaba;

(cuando) el SUB-lugal ${ }^{101}$

97 ninda-š-ill ${ }_{2}$-la: lit. «pan de aquel que alza la mano (orante)»; otra posible interpretación es nig ${ }_{2}$-su-ill 2 -la "propiedades entregadas». En cualquiera de los dos casos, dista de estar claro el sentido de este «abuso».

98 Interpretación de P. Steinkeller, "On the Reading and Meaning of a-ZAR-la», Revue d'Assyriologie 72 (1978) 74 n. 4.

99 Sobre la lectura $\mathrm{E}_{2}, \mathrm{MI}_{2}=a_{x^{\prime}}$, ef. D.O. Edzard, «Soziale 'Reformen' im Zweistromland bis ca. 1600 v. Chr: Realität oder literarischer Topos?», Acta Antiqua Academiae Scientiarum Hungaricae 22 (1974) 148 n. 15.

100 Para la interpretación de este importante pasaje y del verbo $\mathrm{za}_{3}{ }_{3}-\mathrm{us}_{2}$, v. F. Pomponio, «Urukagina 4 VII 11 and an Administrative Term from the Ebla Texts», Journal of Cimeiform Studies 36 (1984) 96-100: «If the equivalence zà-ús $=$ "pll is correct, the verbal form of Ukg 4 vii 11 [= Ukg 5:vi.31] could carry the connotation 'to supervise, to intervene', and the passage in question could then be translated 'in the énsi's palace and in the énsi's fields ... interferences were commited', thus referring to administrative disorders and unlawful interventions by unspecified individuals. These general abuses would have united, not opposed, the é-énsi, the é-É.Mí, and the é-nam-dumu and their respective fields" (pp. 99-100).

101 ŚUB-lugal (vi.37, x.20, xi.2,16): lit. «subordinado al rey/señor»; sobre esta categoría de personas, de características no definidas, v. bibliografía en Steible, Die altsumerischen ... II, p. 150; J.S. Cooper, Presargonic Inscriptions ..., p. 73 añade que se trata de un trabajador libre a quien se ha dado una parcela de campo como compensación parcial por su trabajo. 


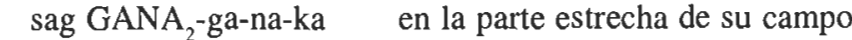

$2 \mathrm{pu}_{2}-\mathrm{ni} \mathrm{i}_{3}-\mathrm{du}_{3}$

3 igi-nu-du

4 ba-dab

5 a-muš-du

6 GANA $_{2}$-ga gal -la-a

7 igi-nu-du 8

8 ba-dab

$9 \quad$ pi-lu ${ }_{5}$-da

10 ud-bi-ta

11 e-me-am ${ }_{6}{ }^{103}$

12 ud d Nin-gir - su

13 ur-sag ${ }^{\mathrm{E}} \mathrm{En}-\mathrm{lil}_{2}-\mathrm{la}_{2}-\mathrm{ke}_{4}$

14 Uru-KA-gi-na-ra

15 nam-lugal

construía su pozo,

al igi-nu-du ${ }_{8}{ }^{102}$

cogía (para el trabajo),

(y también para los trabajos de) los canales de irrigación que tenían lugar en el campo

al igi-nu-du

se cogía.

Las normas

de los tiempos anteriores

eran estas.

Cuando Ningirsu,

héroe de Enlil,

a UruKAgina

16 Lagaš $^{\mathrm{ki}}$

17 e-na-sum-ma-a

la realeza

de Lagaš

le dio,

$18 \stackrel{\text { sa }}{3} l_{2}-36000-t a$

19 šu-ni e-ma-ta-dab - -ba-a

20 nam-tar-ra

(cuando) entre 36000 hombres

su mano cogió,

el destino

21 ud-bi-ta

22 e-še ${ }_{3}$-gar

23 inim lugal-ni

de tiempos anteriores

estableción ${ }^{104}$,

las órdenes que su señor

24 Nin-gir ${ }_{2}-\mathrm{su}^{\mathrm{ke}} \mathrm{k}_{4}$

Ningirsu

25 e-na-dug -ga $^{-}$

le había dado

26 ba-dab

comprendio:

$27 \quad \mathrm{ma}_{2}-\mathrm{ta}$

de(l control de) los barcos

$28 \quad \mathrm{lu}_{2}-\mathrm{ma}_{2}-\mathrm{lah}_{5}$

29 e-ta-šub

30 anše-ta

al «hombre de la barca»

apartó;

de( 1 control de) los asnos

31 udu-ta

$32 \quad u_{2}$-du-bi

33 e-ta-šub

$34 \mathrm{u}_{3}-\mathrm{sar} \mathrm{u}_{2}-\mathrm{mu}_{30}$-ta

(y) de las ovejas

al correspondiente administrador de los rebaños

apartó;

35 enku

de ......

al supervisor de la pesca

apartó;

de las contribuciones de cebada

2 se gub-ba

3 gudu 4 -ge-ne-ta

de los sacerdotes-gudug

102 igi-nu-du $($ vii.3,7, x.1): lit. «ciego»; sobre esta categoría de personas, de características no definidas, v. bibliografía en Steible, Die altsumerischen ... II, pp. 150-151; J.S. Cooper, Presargonic Inscriptions ..., p. 73 añade que se trata de un trabajador no-libre de estatus inferior al del SUB-lugal.

103 Var. Ukg 4:vii.28: e-me-a.

104 Una interpretación diferente del verbo gar con prefijo -še ${ }_{3}^{-}$, que alteraría gran parte del sentido del texto, es la propuesta por B. Foster, «A new Look ...., p. 136, quien traduce la forma verbal e-še ${ }_{3}$ gar como «rechazó». 


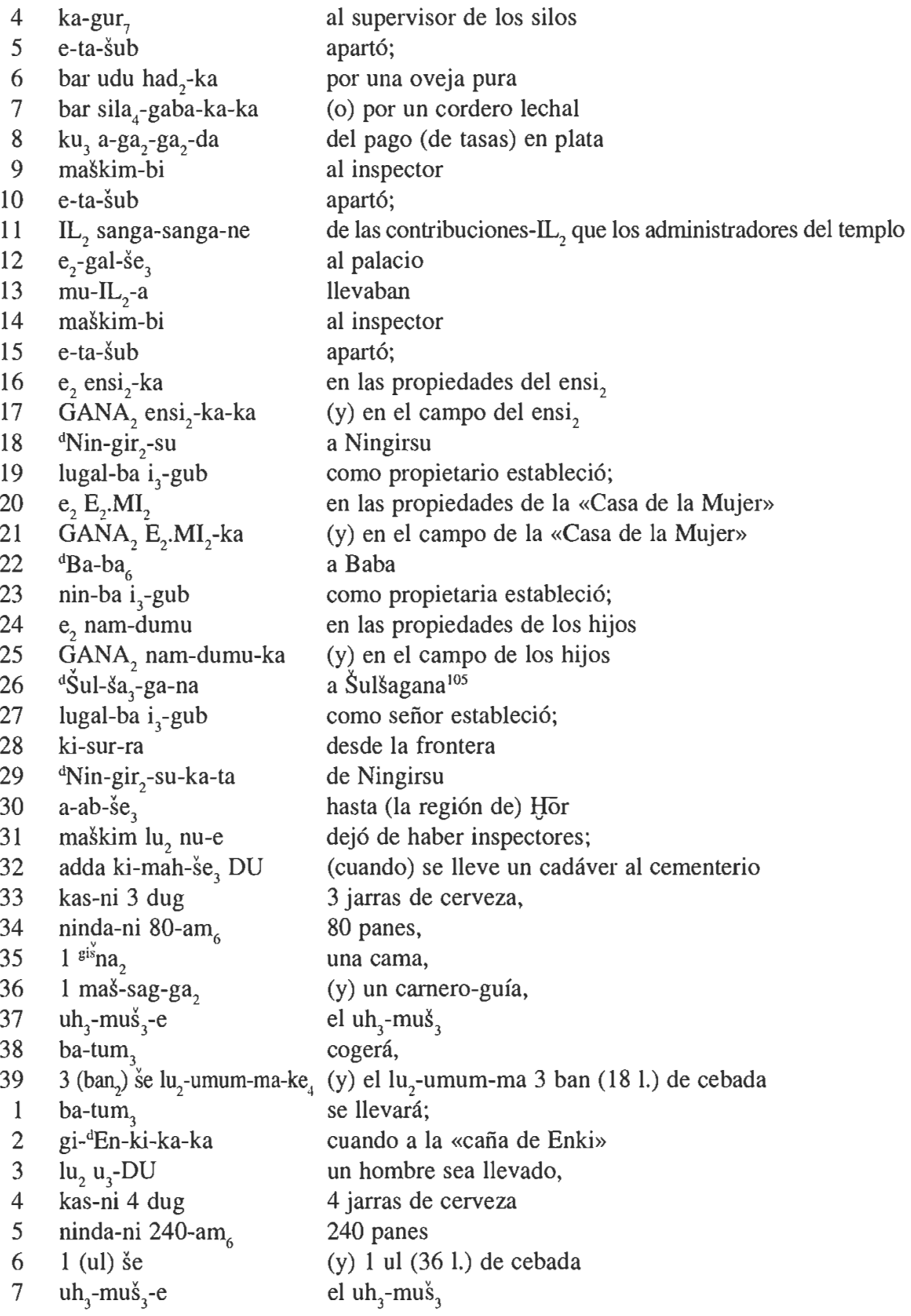

105 Hijo del dios Ningirsu y su esposa Baba. 


\begin{tabular}{|c|c|c|}
\hline 8 & ba-tum ${ }_{3}$ & se llevará, \\
\hline 9 & $3\left(\right.$ ban $\left._{2}\right)$ še lu $u_{2}$-umum-ma-ke & (y) el lu $u_{2}$-umum-ma 3 ban (18 l.) de cebada \\
\hline 10 & ba-tum ${ }_{3}$ & se llevará; \\
\hline 11 & $1 \mathrm{nig}_{2}$-sag-la $\mathrm{a}_{2}-\mathrm{MI}_{2}$ & un tocado de mujer \\
\hline 12 & 1 sila $_{3}$ ir-nun & (y) 1 sila (1 l.) de «perfume de primera calidad» \\
\hline 13 & nin-dingir-re $e_{2}$ & la sacerdotisa-nin-dingir ${ }^{106}$ \\
\hline 14 & ba-tum $_{3}$ & se llevará; \\
\hline 15 & 420 ninda-durun-durun-na & 420 panes secos (?) \\
\hline 16 & ninda gub-ba-am 6 & son los panes de la contribución, \\
\hline 17 & 40 ninda-kum 2 & 40 panes calientes \\
\hline 18 & ka-gub-ba-am 6 & son los panes para la cena (?), \\
\hline 19 & 10 ninda-kum ${ }_{2}$ & 10 panes calientes \\
\hline 20 & ninda-banšur-ra-kam & son los panes de mesa, \\
\hline 21 & 5 ninda-lu ${ }_{2}-z i-g a-k a$ & 5 panes del «hombre del bando» ${ }^{107}$ \\
\hline 22 & 2 kas-LAK.449 $1 \mathrm{sa}_{2}-\mathrm{dug}_{4}$ & 2 ánforas (y) una vasija-sa-dug ${ }_{4}$ de cerveza \\
\hline 23 & gala & es (la ración) del cantante de lamentaciones \\
\hline 24 & $\mathrm{Gir}_{2}-\mathrm{su}^{\mathrm{ki}}-\mathrm{kam}$ & de Girsu; \\
\hline 25 & 490 ninda & 490 panes, \\
\hline 26 & 2 kas-LAK.449 $1 \mathrm{sa}_{2}-\mathrm{dug}_{4}$ & 2 ánforas (y) una vasija-sa-dug ${ }_{4}$ de cerveza \\
\hline 27 & gala & es (la ración) del cantante de lamentaciones \\
\hline 28 & Lagašk $^{k}$-kam & de Lagaš; \\
\hline 29 & 406 ninda & 406 panes, \\
\hline 30 & 1 kas-LAK.449 $1 \mathrm{sa}_{2}-$ dug $_{4}$ & un ánfora y una vasija-sa-dug de cerveza \\
\hline 31 & gala-am ${ }_{6}$ & es (la ración) de los (otros) cantantes de lamentaciones; \\
\hline 32 & 250 ninda & 250 panes \\
\hline 33 & 1 kas-LAK.449 & (y) 1 ánfora de cerveza \\
\hline 34 & nam-um-ma-me 108 & es (la ración) de las ancianas ${ }^{109}$; \\
\hline 35 & 180 ninda & 180 panes \\
\hline 36 & 1 kas-LAK.449 & (y) un ánfora de cerveza \\
\hline 37 & $\mathrm{ABxA} \breve{S}_{2} \cdot \mathrm{IGI}{ }^{110}$ & es (la ración) de los ancianos ${ }^{111}$ \\
\hline
\end{tabular}

106 Sobre este tipo de sacerdotisas, v. bibliografía en Steible, Die altsunerischen ... II, p. 152.

107 Tipo de pan, de características desconocidas, que forma parte de lo destinado a los cantantes de lamentaciones de Girsu (Steible, Die altsumerischen ... II, p. 153).

108 Var. Ukg 4:x,33: nam-um-ma-am.

109 El término (nam-)um-ma significa lit. «mujer anciana», aunque en este caso se suele interpretar como "plañidera» por su relación con um-ma-er, (lit, «anciana que llora») en F. Allotte de la Fuÿe, Documents ..., n 159:i.3 (Steible, Die altsumerischen ... II, p. I54).

110 Var. Ukg 4:x.36: UNU.IGI.

111 Sobre el término ABxAŠ , tanto en su acepción de «anciano» como en la de «testigo», v. I.J. Gelb, «šîbût kušurrā'im, 'Witnesses of the Indemnity'», Journal of Near Eastern Studies 43 (1984) 263-276, e I.J. Gelb - P. Steinkeller - R.M. Whiting, Earliest Land Tenure Systems in the Near East: Ancient Kudurrus, Oriental Institute

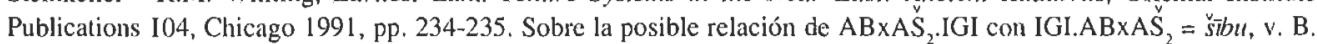
Hruška, «Die innere Struktur ...», p. 113 y, en contra, J. Bauer, «Altsumerische Beiträge ...», p. 8 n. 25. «Ancianos» y «ancianas» aparecen en este pasaje (ix.15-x.10) con motivo de la regulación de las tarifas por una ceremonia de lamentación, no mencionada en la descripción de los abusos; estos personajes aparecen, asimismo, durante el III mil. a.C., en muchos otros textos y circunstancias aún no estudiadas pormenorizadamente. 


\begin{tabular}{|c|c|c|}
\hline & 38 & NINA $^{k i}$-na-me \\
\hline $\mathbf{x}$ & 1 & igi-nu-du \\
\hline & 2 & zar -ra-a gub-ba \\
\hline & 3 & ninda-ka gub-ba-ni 1-am \\
\hline & 4 & 5 ninda-gi $i_{6}-b a-a-k a-n i$ \\
\hline & 5 & 1 ninda-UD.SA ${ }_{2} \cdot A-k a-n i^{112}$ \\
\hline & 6 & 6 ninda-gi ${ }_{6}$-an-na-ka-ni \\
\hline & 7 & 60 ninda \\
\hline & 8 & 1 kas-LAK.449 \\
\hline & 9 & $3\left(\mathrm{ban}_{2}\right) \mathrm{se}$ \\
\hline & 10 & $\mathrm{lu}_{2}$ sag-bur-re, ak-da-kam \\
\hline & 11 & addir a-bul - -la \\
\hline & 12 & guruŝ-MIN-ka \\
\hline & 13 & $\operatorname{inim} i_{3}-g i_{4}$ \\
\hline & 14 & giš-kin-ti \\
\hline & 15 & ninda-šu-il ${ }_{2}-\mathrm{la}-\mathrm{ba}$ \\
\hline & 16 & $\operatorname{inim} i_{3}-g i_{4}^{2}$ \\
\hline & 17 & sanga-GAR \\
\hline & 18 & kiri $_{6}$ ama- $\mathrm{uku}_{2}-\mathrm{ra}_{2}$ \\
\hline & 19 & nu-lah \\
\hline & 20 & SUUB-lugal-ra \\
\hline & 21 & anše-ša ${ }_{6}-g a$ \\
\hline & 22 & $\mathrm{u}_{3}$-na-tu \\
\hline & 23 & ugula-ne, \\
\hline & 24 & $g a-s ̌ e_{3}-s a_{10}$ \\
\hline & 25 & $\mathrm{u}_{3}$-na-dug $\mathrm{g}_{4}$ \\
\hline & 26 & $\mathrm{u}_{3}$-da mu-se $-\mathrm{sa}_{10}-\mathrm{sa}_{10} 114$ \\
\hline & 27 & $k u_{3} \mathrm{sa}_{3}-g a_{2} a-s a_{6}-g a$ \\
\hline & 28 & $\mathrm{la}_{2}-\mathrm{ma}$ \\
\hline & 29 & $\mathrm{u}_{3}$-na-dug \\
\hline & 30 & ud nu-še $-\mathrm{s}_{3} \mathrm{~s}_{10}-\mathrm{sa} \mathrm{a}_{10}-\mathrm{a}^{-\mathrm{a}} 115$ \\
\hline & 31 & ugula $\mathrm{AB}_{2} \cdot{ }_{\mathrm{S}} \mathrm{A}_{3}$-bi \\
\hline & 32 & na-na-tag-ge \\
\hline $\mathbf{x i}$ & 1 & $\mathrm{e}_{2} \operatorname{lu}_{\mathrm{v}}-\mathrm{gu}-\mathrm{la}-\mathrm{ke}_{4}$ \\
\hline & 2 & $\mathrm{e}_{2}$ SUB-lugal-ka \\
\hline & 3 & $\mathrm{ab}-\mathrm{us}_{2}-\mathrm{sa}{ }^{116}$ \\
\hline & 4 & $l u_{2}-g u-l a-b i$ \\
\hline
\end{tabular}

de NINA;

para el igi-nu-du de servicio en el ...

(su ración es de) un pan para la cena (?),

5 panes para el amanecer (?),

un pan para el mediodía (?)

(y) 6 panes para la noche;

60 panes,

un ánfora de cerveza

(y) 3 ban (181.) de cebada

(es la ración) para los que trabajan como sag-bur ${ }^{113}$;

la tarifa de 'la puerta de la ciudad'

para las parejas de obreros

él (UruKAgina) revocó,

(y) para los artesanos

el ninda-šu-il $-\mathrm{la}$

revocó;

los sacerdotes-GAR

el jardín del ama-uku

dejaron de saquear;

(cuando) a un ŠUB-lugal

un asno de buena calidad

le nazca,

(y) su supervisor

«te lo quiero comprar»

le diga,

«si lo quieres comprar

el precio que satisfaga a mi corazón

págame»

(si el ŠUB-lugal) le responde,

(o) si no se lo quiere vender,

el supervisor coléricamente

no le golpeará;

cuando la propiedad de un 'hombre grande'

con la propiedad de un ŠUB-lugal

limite,

y ese 'hombre grande'

112 Var. Ukg 4:xi.5: ninda-UD.SA ${ }_{2}$-ka-ni.

113 sag-bur = ac. abru [CAD A/1 p. 64 s.v. abru C: «(a priestly official of low standing)»], assinnu [CAD A/2 p. 341 s.v. assinnu; "(a member of the cultic personnel of Istar)»]; esta última profesión se encuentra en contexto con el cantante de lamentaciones (J.S. Cooper, Presargonic Inscriptions ..., p. 74), al igual que ocurre en nuestro párrafo ix.15x. 10 .

114 Var. Ukg 4:xi.25: $\mathrm{u}_{4}$-da mu-se ${ }_{3}-\mathrm{sa}_{10}-\mathrm{sa}_{10}$.

115 Var. Ukg 4:29: ud nu- $\mathrm{Se}_{3}-\mathrm{Sa}_{10}-\mathrm{Sa}_{10}$ 


\begin{tabular}{|c|c|}
\hline 5 & $g a-s e_{3}-s a_{10}$ \\
\hline 6 & $\mathrm{u}_{3}$-na-dug \\
\hline 7 & $\mathrm{u}_{3}-\mathrm{da} 117$ \\
\hline 8 & $m u-s e_{3}-s a_{10}-s a_{10}$ \\
\hline 9 & $k u_{3} \mathrm{ša}_{3}-g a_{2} a-s a_{6}-g a$ \\
\hline 10 & $\mathrm{la}_{2}-\mathrm{ma}$ \\
\hline 11 & $\mathrm{e}_{2}-\mathrm{mu}$ pisan $_{3}$-nam \\
\hline 12 & se si-ma-ni \\
\hline 13 & $u_{3}$-na-dug 4 \\
\hline 14 & $\mathrm{u}_{3}-\mathrm{da}$ nu-se $\mathrm{e}_{3}-\mathrm{sa}_{10}-\mathrm{sa}_{10}{ }^{118}$ \\
\hline 15 & $\operatorname{lu}_{2}-g u-l a-b i$ \\
\hline 16 & ŚUB-lugal-ra \\
\hline 17 & $\mathrm{AB}_{2} \cdot \mathrm{SA}_{3}-\mathrm{bi}$ \\
\hline 18 & na-na-tag-ge \\
\hline 19 & $\mathrm{i}_{3}-\mathrm{dug}_{4}$ \\
\hline 20 & dumu Lagaški \\
\hline 21 & $\mathrm{ur}_{5}$-ra ti-la \\
\hline 22 & gur gub-ba \\
\hline 23 & še si-ga \\
\hline
\end{tabular}

$24 \quad$ nig $_{2}$ zuh-a

25 sag giš ra-a

$26 \mathrm{e}_{2}$-E $\dot{S}_{2}-\mathrm{bi}$

27 e-luh

28 ama-gi 4 -bi

29 e-gar

30 nu-siki nu-ma- $\{$ nu- $\}$ su ${ }^{120}$

$31 \quad \mathrm{lu}_{2}-\mathrm{a}_{2}$-tuku

xii 1 nu-na-ga $-\mathrm{ga}_{2}-\mathrm{a}$

2 dNin-gir 2 -su-da

3 Uru-KA-gi-na-ke

4 inim-bi ka e-da-keš

5 ša ${ }_{3}$ mu-ba-ka

$6 \mathrm{id}_{2}$-tur- $\mathrm{Gir}_{2}-\mathrm{su}^{\mathrm{ki}}-$

$7 \quad \mathrm{i}_{3}$-tuku-a

8 "Nin-gir ${ }_{2}$-su-ra

9 al mu-na-du

10 mu ud-bi-ta-bi «quiero comprártelo»

le diga,

«si

la quieres comprar

el precio que satisfaga a mi corazón

págame,

mi casa es un gran recipiente-pisan ${ }_{3}$,

¡llénamelo de cebada!»

(si el ŠUB-lugal) le responde,

(o) si no se lo quiere vender,

el 'hombre grande'

al ŠUB-lugal

coléricamente

no le golpeará.

(Esto es lo que UruKAgina) ha dicho.

A los ciudadanos de Lagaš:

al que estaba endeudado,

al que había establecido una medida-gur (falsa),

al que había llenado (inapropiadamente una medida-gur con) cebada,

al ladrón

(y) al asesino,

su prisión

él la ha limpiado (de ellos);

su libertad

él ha dispuesto" 11 .

Que el huérfano y la viuda

al poderoso

no le sean entregados

con Ningirsu

UruKAgina

ha acordado.

En ese año

el pequeño canal que Girsu

tiene

a Ningirsu

le ha excavado;

el nombre que desde ese día

116 Var. Ukg 4:xi.34: a(sobre $A B)$-us ${ }_{2}$-sa.

117 Var. Ukg 4:xi.38: $u_{4}$-da.

118 Var. Ukg 4:xii.7: $\mathrm{u}_{4}$-da nu-se $\mathrm{v}_{3}-\mathrm{sa}_{10}-\mathrm{sa}_{10}$.

119 xi.20-29: la interpretación aquí propuesta para este importante pasaje sigue a P. Steinkeller, «The Reforms of UruKAgina ...», pp. 227-232.

120 Var. Ukg 4:xii.23: nu-ma-su. 


\begin{tabular}{|c|c|c|}
\hline 11 & e-še $e_{3}$-gar & lleva, \\
\hline 12 & 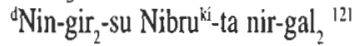 & «Ningirsu-es-Soberano-ante Nippur», \\
\hline 13 & Uru-KA-gi-na-ke ${ }_{4}$ & UruKAgina \\
\hline 14 & mu mu-na-sa & le ha dado; \\
\hline 15 & $\mathrm{id}_{2}-\mathrm{NINA}^{\mathrm{ki}}$-gin-a & hasta el «Canal-que-fluye- hacia-NINA» \\
\hline 16 & mu-na-ni-la ${ }_{2}$ & él lo hizo llegar; \\
\hline 17 & $\mathrm{id}_{2} \mathrm{ku}_{3}-\mathrm{ga}-\mathrm{am}_{6}$ & el canal es puro, \\
\hline 18 & ša ${ }_{3}$-bi dadag-ga-am 6 & su lecho es claro; \\
\hline 19 & "Nanše & ¡que a Nanše \\
\hline 20 & a-zal-le & agua corriente \\
\hline 21 & he $_{2}$-na-tum 3 & le traiga! ${ }^{122}$ \\
\hline
\end{tabular}

\section{BIBLIOGRAFÍA}

ALLOTTE DE LA FUŸE, F., Documents présargoniques, París 1908-1920.

ASHER-GREVE, J.M., Frauen in altsumerischer Zeit, Bibliotheca Mesopotamica 18, Malibu 1985.

BAUER, J., «Zum Totenkult im altsumerischen Lagasch», Zeitschrift der Deutschen Morgenländischen Gesellschaft. Suppplementa I (1969) 107-114.

BAUER, J., Altsumerische Wirtschaftstexte aus Lagasch, Roma 1972.

BAUER, J., «Altsumerische Beiträge (7-9)», Die Welt des Orients 9 (1977-78) 1-9.

BEHRENS, H. - STEIBLE, H., Glossar zu den altsumerischen Bau- und Weihinschriften, Freiburger Altorientalische Studien 6, Wiesbaden 1983.

CARROUÉ, F., «Le 'Cours-d'Eau-Allant-à-NINA ki'», Acta Sumerologica 8 (1986) 13-57.

COOPER, J.S., Reconstructing History from Ancient Inscriptions: the Lagash-Umma Border Conflict, Sources from the Ancient Near East 2/1, Malibu 1983.

COOPER, J.S., «Medium and Message: Inscribed Clay Cones and Vessels from Presargonic Sumer», Revue d'Assyriologie 79 (1985) 97-114.

COOPER, J.S., Presargonic Inscriptions. Sumerian and Akkadian Royal Inscriptions I, New Haven 1986.

DEIMEL, A., «Die Reformtexte Urukaginas», Orientalia SP 2 (1920) 6-31.

DEIMEL, A., Sumerische Tempelwirtschaft zur Zeit Urukaginas und seiner Vorgänger, Analecta Orientalia 2, Roma 1931.

DIAKONOFF, I.M., «The Rise of the Despotic State in Ancient Mesopotamia», en I.M. Diakonoff (ed.), Ancient Mesopotamia: Socio-Economic History. A Collection of Studies by Soviet Scholars, Moscú 1969, pp. 173-203 (art. aparecido en 1956).

DIAKONOFF, I.M., «Some Remarks on the 'Reforms' of Urukagina», Revue d'Assyriologie 52 (1958) $1-15$.

EDZARD, D.O., «Soziale 'Reformen' im Zweistromland bis ca. 1600 v. Chr.: Realität oder literarischer Topos?», Acta Antiqua Academiae Scientiarum Hungaricae 22 (1974) 145-156.

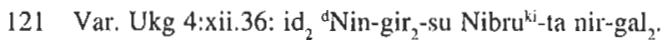

122 xii.5-21: sobre este pasaje, v. Carroué, «Le 'Cours-d'Eau-...», pp. 18-19. 
EDZARD, D.O., «Irikagina (Urukagina)», Aula Orientalis $9(1991)=F s$. M. Civil, pp. 77-79. EDZARD, D.O. - FARBER, G., Die Orts- und Gewässernamen der 3. Dynastie von Ur, Répertoire Géographique des Textes Cunéiformes 2, Wiesbaden 1974.

EDZARD, D.O. - FARBER, G. - SOLLBERGER, E., Die Orts- und Gewässernamen der präsargonischen und sargonischen Zeit, Répertoire Géographique des Textes Cunéiformes 1, Wiesbaden 1977.

ELLIS, R.S., Foundation Deposits in Ancient Mesopotamia, Yale Near Eastern Researches 2, New Haven - Londres 1968.

FALKENSTEIN, A., «La Cité-Temple Sumérienne», Cahiers de l'Histoire Mondiale 1 (1954) 784-814. Traducción al inglés, por M. de J. Ellis, en Monographs on the Ancient Near East 1/1, Malibu 1974.

FÖRTSCH, W., Altbabylonische Wirtschaftstexte aus der Zeit Lugalanda's und Urukagina's, Vorderasiatische Schriftdenkmäler der Königlichen Museen zu Berlin 14/I, Leipzig 1916.

FOSTER, B., "A New Look at the Sumerian Temple State», Journal of the Economic and Social History of the Orient 24 (1981) 225-241.

FOXVOG, D. A., «A New Lagaš Text Bearing on Uruinimgina's Reforms», Journal of Cuneuform Studies 46 (1994) 11-15.

GELB, I.J., «Household and Family in Early Mesopotamia», en E. Lipinski (ed.), State and Temple Economy in the Ancient Near East I, Orientalia Lovaniensia Analecta 5, Leuven 1979, pp. 1-97.

GELB, I.J., «šîbût kušurrā'im, 'Witnesses of the Indemnity'», Journal of Near Eastern Studies 43 (1984) 263-276.

GELB, I.J. - STEINKELLER, P. - WHITING, R.M., Earliest Land Tenure Systems in the Near East: Ancient Kudurrus, Oriental Institute Publications 104, Chicago 1991.

GENOUILLAC, H. de, Tablettes sumériennes archaiques, París 1909.

HALLO, W.W., Early Mesopotamian Royal Titles: A Philologic and Historical Analysis, American Oriental Series 43, New Haven 1957.

HEIMPEL, W., «Herrentum und Königtum im vor- und frühgeschichtlichen Alten Orient», Zeitschrift fiir Assyriologie 82 (1992) 4-21.

HRUŠKA, B., «Die Reformtexte Urukaginas. Der verspätete Versuch einer Konsolidierung des Stadtstaates von Lagaš», Le Palais et la Royauté (Archéologie et Civilisation) $=X X^{c}$ Réncontre Assyriologique Internationale, Paris 1971, pp. 151-162.

HRUŠKA, B., "Die innere Struktur der Reformtexte Urukaginas von Lagaš», Archiv Orientalni 41 (1975) 4-13 y 104-132.

HRUŠKA, B., «Die Bewässerungsanlagen in den altsumerischen Königsinschriften von Lagaš», Bulletin on Sumerian Agriculture 4 (1988) 61-72.

JACOBSEN, Th., «Early Political Development in Mesopotamia», Zeitschrift für Assyriologie 18 (1957) 91-140.

JACOBSEN, Th., «The Term Ensí», Aula Orientalis 9 (1991) = Fs. M. Civil, pp. 113-121.

KOBAYASHI, T., «The ki-a-nag of Enentarzi», Orient 21 (1985) 10-30.

KRAMER, S.N., The Sumerians. Their History, Culture and Character, Chicago 1963.

KRAMER, S.N., «The Ur-Nammu Code: Who was its Author?», Orientalia NS 52 (1983) 453456.

LAMBERT, M., «Les 'reformes' d'Urukagina», Revue d'Assyriologie 50 (1956) 169-184.

LAMBERT, M., «Le quartier Lagash», Rivista degli Studi Orientali 32 (1957) 123-143. 
LAMBERT, M., «La guerre entre Urukagina et Lugalzaggesi», Rivista degli Studi Orientali 41 (1966) 29-66.

LAMBERT, M., «L'expansion de Lagash au temps d'Entemena», Rivista degli Studi Orientali 47 (1972) 1-22.

LAMBERT, M., «Recherches sur les réformes d'Urukagina», Orientalia NS 44 (1975) 22-51.

LAMBERT, W.G., «The Reading of the Name uru.KA.gi.na», Orientalia NS 39 (1970) 419.

LAMBERT, W.G., «The Reading of Uru-KA-gi-na Again», Aula Orientalis 10 (1992) 256-258.

LIVERANI, M., Antico Oriente. Storia, società, economia, Roma-Bari 1988.

MAEDA, T., «'King of Kish' in Pre-Sargonic Sumer», Orient 17 (1981) 1-17.

MAEDA, T., «Work Concerning Irrigation Canals in Pre-Sargonic Lagash», Acta Sumerologica 6 (1984) 33-53.

MAEKAWA, K., «The Development of the É.MÍ in Lagaš during Early Dynastic III», Mesopotamia 8-9 (1973-74) 77-144.

MICHALOWSKI, P. - WALKER, C.B.F., «A new Sumerian 'Law Code'», en H. Behrens - D. Loding - M.T. Roth (edd.), DUMU-É-DUB-BA-A, Studies in Honor of Ake Sjöberg, Occasional Publications of the Samuel Noah Kramer Fund 11, Philadelphia 1989, pp. 383-396.

NIKOLSKIJ, M.V., Dokumenty chozjajstvennoj ot Četnosti drevnêjšej epochi chaldei iz sobranija N.P. Lichačeva. I, Drevnosti Vostočnyja 3, S. Petersburgo 1908.

NISSEN, H.J., The Early History of the Ancient Near East. 9000-2000 B.C., Chicago 1988 [Darmstadt 1983].

PETTINATO, G., «I $\mathrm{I}_{7}$ Idigna-ta $\mathrm{I}_{7}$-nun-še $\mathrm{e}_{3}$. Il conflitto tra Lagaš ed Umma per la 'Frontiera Divina' e la sua soluzione durante la Terza Dinastia di Ur', Mesopotamia 5-6 (1970-71) 281-320.

PETTINATO, G., I Sumeri, Milán 1992.

POMPONIO, F., «Urukagina 4 VII 11 and an Administrative Term from the Ebla Texts», Journal of Cuneiform Studies 36 (1984) 96-100.

POMPONIO, F., La prosopografia dei testi presargonici di Fara, Studi Semitici NS 3, Roma 1987.

ROSENGARTEN, Y., «La notion de souveraineté divine», Revue d'Histoire des Religions 156 (1959) 129-146.

ROSENGARTEN, Y., Le concept sumérien de consommation dans la vie économique et religieuse, París 1960.

SARZEC, E. de - HEUZEY, L., Découvertes en Chaldée, París 1884-1912.

SCHNEIDER, N., Die sumerische Tempelstadt, Essen 1920.

SELZ, G.B., Die altsumerischen Wirtschaftsurkunden der Ermitage zu Leningrad, Freiburger Altorientalische Studien 15/1, Stuttgart 1989.

SELZ, G.B., «Studies in Early Syncretism: The Development of the Pantheon in Laggas. Examples for Inner-Sumerian Syncretism», Acta Sumerologica 12 (1990) 111-142.

SOLLBERGER, E., Corpus des Inscriptions «Royales» Présargoniques de Lagash, Genève 1956

SOLLBERGER, E., «L'opposition au pays de Sumer et Akkad», en A. Finet (ed.), La Voix de l'Opposition en Mésopotamie, Bruxelles 1973, pp. 28-36.

STEIBLE, H., Die altsumerischen Bau- und Weihinschriften I-II, Freiburger Altorientalische Studien 5, Wiesbaden 1982.

STEINER, G., «Altorientalische 'Reichs'-Vorstellungen im 3. Jahrtausend v.Chr.», en M.T. 
Larsen (ed.), Power and Propaganda. A Symposium on Ancient Empires, Mesopotamia (Copenhagen Studies in Assyriology) 7, Copenhagen 1979, pp. 125-143.

STEINER, G., «Der Grenzvertrag zwischen Lagaš und Umma», Acta Sumerologica 8 (1986) 219-300.

STEINKELLER, P., «On the Reading and Meaning of a-ZAR-la», Revue d'Assyriologie 72 (1978) 73-76.

STEINKELLER, P., «More Evidence for the Reading bul of LAGABxSUM», Revue d'Assyriologie 73 (1979) 91-92; «Other Additions to RA 72 pp. 73-76», Revue d'Assyriologie 73 (1979) 92-93.

STEINKELLER, P., «The Renting of Fields in Early Mesopotamia and the Development of the Concept of 'Interest' in Sumerian", Journal of the Economic and Social History of the Orient 24 (1981) 132-134.

STEINKELLER, P., «The Administrative and Economic Organization of the Ur III State: The Core and the Periphery", en McG. Gibson - R.D. Biggs (edd.), The Organization of Power. Aspects of Bureaucracy in the Ancient Near East, Studies in Ancient Oriental Civilization 46, Chicago 1987, pp. 19-41.

STEINKELLER, P., "The Reforms of UruKAgina and an Early Sumerian Term for 'Prison'», Aula Orientalis $9(1991)=$ Fs. M. Civil, pp. 227-233.

STEINKELLER, P., «Early Political Development in Mesopotamia and the Origins of the Sargonic Empire», en M. Liverani (ed.), Akkad: The First World Empire: Structure, Ideology, Traditions, History of the Ancient Near East / Studies 5, Padua 1993, pp. 107-129.

STRUVE, V.V., "The Problem of the Genesis, Development and Disintegration of the Slave Societies in the Ancient Orient», en I.M. Diakonoff (ed.), Ancient Mesopotamia: SocioEconomic History. A Collection of Studies by Soviet Scholars, Moscú 1969, pp. 17-70 (art. aparecido en 1933).

THOMSEN, M.-L., The Sumerian Language. An Introduction to its History and Grammatical Structure, Mesopotamia (Copenhagen Studies in Assyriology) 10, Copenhagen 1984.

THUREAU-DANGIN, F. , Recueil de tablettes chaldéennes, París 1903.

THUREAU-DANGIN, F., Die sumerischen und akkadischen Königsinschriften, Leipzig 1907. TYUMENEV, A.I., «The Working Personnel on the Estate of the Temple of ${ }^{\text {Ba-Ú in Lagas }}$ During the Period of Lugalanda and Urukagina (25th-24th cent. B.C.)», en I.M. Diakonoff (ed.), Ancient Mesopotamia: Socio-Economic History. A Collection of Studies by Soviet Scholars, Moscú 1969, pp. 88-126 (art. aparecido en 1954).

VANSTIPHOUT, H., «Political Ideology in Early Sumer», Orientalia Lovaniensia Periodica 1 (1970) 1-38.

WAETZOLDT, H., «Zu den Strandverschiebungen am Persischen Golf und den Bezeichnungen des Hōors», en Rupert Carola, Sonderheft Strandverschiebungen, Heidelberg 1981, pp. 168169.

WESTENHOLZ, A., «Diplomatic and Commercial Aspects of Temple Offerings as Illustrated

by a Newly Discovered Text», Iraq 39 (1977) 19-21.

YANG, Z., «King of Justice», Aula Orientalis 9 (1991) = Fs. M. Civil, pp. 243-249.

YILDIZ, F., «A Tablet of Codex U1'-nammu from Sippar», Orientalia NS 50 (1981) 87-97. 